\title{
SUBGRAPHS OF RANDOM GRAPHS
}

BY

\author{
D. H. FREMLIN ${ }^{1}$ AND M. TALAGRAND
}

\begin{abstract}
Let $\Delta \subseteq[\omega]^{2}$ be an undirected graph on $\omega$, and let $u \in[0,1]$. Following P. Erdös and A. Hajnal, we write $(\omega, 2, u) \Rightarrow \Delta$ to mean: whenever $E_{I} \subseteq[0,1]$ is a measurable set of Lebesgue measure at least $u$ for every $I \in[\omega]^{2}$, then there is some $t \in[0,1]$ such that $\Delta$ appears in the graph $\Gamma_{t}=\left\{I: t \in E_{I}\right\}$ in the sense that there is a strictly increasing function $f: \omega \rightarrow \omega$ such that $\{f(i), f(j)\} \in \Gamma_{t}$ whenever $\{i, j\}$ $\in \Delta$. We give an algorithm for determining when $(\omega, 2, u) \Rightarrow \Delta$ for finite $\Delta$, and we show that for infinite $\Delta,(\omega, 2, u) \Rightarrow \Delta$ if there is a $v<u$ such that $(\omega, 2, v) \Rightarrow \Delta^{\prime}$ for every finite $\Delta^{\prime} \subseteq \Delta$. Our results depend on a new condition, expressed in terms of measures on $\beta \omega$, sufficient to imply that $\Delta$ appears in $\Gamma$ (Theorem $2 F$ ), and enable us to identify the extreme points of some convex sets of measures (Theorem $5 \mathrm{H}$ ).
\end{abstract}

Introduction. In [1], P. Erdös and A. Hajnal raised a variety of questions of the following type. Suppose we are given a random process for generating a graph, and we know that the probability of any particular link appearing in the graph is at least $u$, but we are told nothing about the intercorrelations of different links. When can we conclude that certain configurations have positive probability of appearing somewhere in the graph? They give a number of results, both positive and negative, and leave open many problems. In this paper we develop techniques which solve nearly all their problems concerning ordinary graphs on the nonnegative integers, and which appear applicable to many others.

The genesis of this paper was a conversation with P. Erdös, during which he mentioned his long-standing conjectures, here proved as Corollary 4B and (in a strengthened form) as Corollary $4 \mathrm{D}$. The ideas of $\S \S 2$ and 5 were stimulated by a timely lecture by $\mathbf{J}$. L. Krivine, and $3 \mathrm{M}(\mathrm{a})$ was worked out in conversation with $\mathbf{J}$. Saint-Raymond. The rest of $\S 3$ was done in the light of calculations using the facilities of the University of Essex Computing Service. We should also like to acknowledge useful conversations with J. W. Kennedy, B. Bollobas and I. T. Cook.

\section{Formulation of the problems.}

1A. Definitions. We begin with bald statements of the definitions which dominate this paper.

(a) If $X$ is any totally ordered set, write

$$
\begin{aligned}
& X^{2 \uparrow}=\{(x, y): x, y \in X, x<y\}, \\
& X^{\omega \uparrow}=\left\{f: f \in X^{\omega}, f \text { is strictly increasing }\right\} .
\end{aligned}
$$

Received by the editors October 4, 1984.

1980 Mathematics Subject Classification. Primary 60C05; Secondary 05C99.

Key words and phrases. Random graphs, $\beta \omega$, extreme points.

${ }^{1}$ Part of the work of this paper was done while this author held a visiting professorship at the Université Paris VI. 
(b) If $H$ is a relation (i.e. a set of ordered pairs), write

$$
\begin{aligned}
V(H) & =\pi_{1}[H] \cup \pi_{2}[H] \\
& =\{x: \exists y(x, y) \in H \text { or }(y, x) \in H\},
\end{aligned}
$$

and call $V(H)$ the set of vertices of $H$.

(c) If $H$ is a relation and $f$ is a function with $V(H) \subseteq \operatorname{dom}(f)$, write

$$
f^{\rightarrow}(H)=\{(f(x), f(y)):(x, y) \in H\} .
$$

(d) If $\Delta, \Gamma \subseteq \omega^{2}$ we say that $\Delta$ appears in $\Gamma$, and write $\Delta \subseteq \Gamma$, if there is an $f \in \omega^{\omega \uparrow}$ such that $f \rightarrow(\Delta) \subseteq \Gamma$.

(e) If $\Delta, H$ are two relations, we say that $H$ admits $\Delta$, and write $\Delta \preccurlyeq H$, if there is a function $f: V(\Delta) \rightarrow V(H)$ such that $f \rightarrow(\Delta) \subseteq H$.

(f) Write $\mu_{L}$ for Lebesgue measure on $[0,1]$. For $\Delta \subseteq \omega^{2 \uparrow}$ and $u \in[0,1]$, write $(\omega, 2, u) \Rightarrow \Delta$ to mean that whenever $\left\langle E_{i j}\right\rangle_{i<j<\omega}$ is a family of measurable subsets of $[0,1]$ with $\mu_{L} E_{i j} \geqslant u$ for every $i<j$, there is a $t \in[0,1]$ such that $\Delta \subseteq\{(i, j)$ : $\left.t \in E_{i j}\right\}$.

(g) Observe that if $\Delta \subseteq \omega^{2 \uparrow}, 0 \leqslant u \leqslant v \leqslant 1$, and $(\omega, 2, u) \Rightarrow \Delta$, then $(\omega, 2, v) \Rightarrow \Delta$. Consequently, there is a unique $u_{0}=u_{0}(\Delta) \in[0,1]$ such that

$$
\left.(\omega, 2, u) \Rightarrow \Delta \quad \forall u \in] u_{0}, 1\right], \quad(\omega, 2, u) \nRightarrow \Delta \quad \forall u \in\left[0, u_{0}[.\right.
$$

(h) It is convenient to introduce here the names we will use for certain subsets of $\omega^{2 \uparrow}$. If $i<j<\omega$, set

$$
U_{i j}=\left\{\Gamma:(i, j) \in \Gamma \subseteq \omega^{2 \uparrow}\right\}
$$

If $\Delta \subseteq \omega^{2}$, set

$$
T_{\Delta}=\left\{\Gamma: \Delta \subseteq \Gamma \subseteq \omega^{2 \uparrow}\right\}
$$

(so that $T_{\Delta}=\varnothing$ if $\Delta \nsubseteq \omega^{2 \uparrow}$ ),

$$
\begin{aligned}
& S_{\Delta}=\left\{\Gamma: \Delta \subseteq \Gamma \subseteq \omega^{2 \uparrow}\right\}=\bigcup\left\{T_{f \rightarrow(\Delta)}: f \in \omega^{\omega \uparrow}\right\}, \\
& S_{\Delta}^{*}=\left\{\Gamma: \Delta \preccurlyeq \Gamma \subseteq \omega^{2 \uparrow}\right\}=\bigcup\left\{T_{f^{\rightarrow(\Delta)}}: f \in \omega^{\omega}\right\} .
\end{aligned}
$$

(i) Finally, we give a definition which will be used in $2 \mathrm{H}$ and will dominate $\S 3$. If $H$ is a finite relation, then its value $v(H)$ is

$$
\sup \left\{\sum_{(x, y) \in H} \alpha_{x} \alpha_{y}: \alpha_{x} \geqslant 0 \forall x \in V(H), \sum_{x \in V(H)} \alpha_{x} \leqslant 1\right\} .
$$

Observe that this supremum is always attained, and that $0 \leqslant v(H) \leqslant 1$.

1B. The problem. Find $u_{0}(\Delta)$ for a given $\Delta \subseteq \omega^{2 \uparrow}$.

1C. Remarks. (a) The " $(\omega, 2)$ " in " $(\omega, 2, u) \Rightarrow \Delta$ " is redundant for the purposes of this paper. We preserve it in order to maintain links with [1], where various generalizations are studied.

(b) An (undirected) graph on $\omega$ is most naturally regarded as a subset of $[\omega]^{2}$, as is done in [1]. However, we shall find that identifying it with the corresponding subset of $\omega^{2 \uparrow}$ simplifies many of the formulas below. 
(c) In [1], the notation " $(\omega, 2, u) \Rightarrow n$ " is used for what we shall write as " $(\omega, 2, u) \Rightarrow n^{2 \uparrow " ; ~ t h a t ~ i s, ~ t h e ~ c a s e ~} \Delta=\{(i, j): i<j<n\}$, the complete graph on $n$ vertices.

(d) The definition $1 \mathrm{~A}(\mathrm{f})$ seems to be the appropriate starting point for certain generalizations studied in [1]. For our purposes, however, the equivalent form in $1 \mathrm{E}$ is more natural.

1D. Measures on $\mathscr{P} \omega^{2 \uparrow}$. As $\omega^{2 \uparrow}$ is countable, its power set $\mathscr{P} \omega^{2 \uparrow}$ has a natural compact metrizable zero-dimensional topology generated by the sets $U_{i j}$ of $1 \mathrm{~A}(\mathrm{~h})$ and their complements. Let $M_{1}\left(\mathscr{P} \omega^{2 \uparrow}\right)$ be the set of Radon probability measures on $\mathscr{P} \omega^{2 \uparrow}$. Observe that each member of $M_{1}\left(\mathscr{P} \omega^{2 \uparrow}\right)$ is determined by its values on the sets $T_{\Delta}$ of $1 \mathrm{~A}(\mathrm{~h})$. For $\nu \in M_{1}\left(\mathscr{P} \omega^{2 \uparrow}\right)$, write

If $\Delta \subseteq \omega^{2}$, then

$$
w(\nu)=\inf _{i<j<\omega} \nu\left(U_{i j}\right)
$$

$$
\left\{(f, \Gamma): f \in \omega^{\omega \uparrow}, f^{\rightarrow}(\Delta) \subseteq \Gamma\right\} \quad \text { and } \quad\left\{(f, \Gamma): f \in \omega^{\omega}, f^{\rightarrow}(\Delta) \subseteq \Gamma\right\}
$$

are closed sets in $\omega^{\omega} \times \mathscr{P} \omega^{2 \uparrow}$, where $\omega^{\omega}$ is given its usual Polish topology. Consequently $S_{\Delta}$ and $S_{\Delta}^{*}$ are analytic sets in $\mathscr{P} \omega^{2 \uparrow}$ and are measurable for every $\nu \in$ $M_{1}\left(\mathscr{P} \omega^{2 \uparrow}\right)$.

We shall say that a measure $\nu \in M_{1}\left(\mathscr{P} \omega^{2 \uparrow}\right)$ is deletion-invariant if $\nu\left(T_{\Delta}\right)=$ $\nu\left(T_{f \rightarrow(\Delta)}\right)$ for any $\Delta \subseteq \omega^{2 \uparrow}$ and any $f \in \omega^{\omega \uparrow}$; equivalently, if $\nu\left(T_{\Delta}\right)=\nu\left(T_{f \rightarrow(\Delta)}\right)$ whenever $\Delta$ is a finite subset of $\omega^{2 \uparrow}$ and $f$ is a function of the form

$$
f(i)=i \quad \forall i<k, \quad f(i)=i+1 \quad \forall i \geqslant k .
$$

For such a measure we find that, if $\Delta \subseteq \omega^{2 \uparrow}$ is finite,

$$
\nu\left(S_{\Delta}\right)>0 \text { iff } \nu\left(T_{\Delta}\right)>0
$$

To see this, observe that (because $\Delta$ is finite)

$$
S_{\Delta}=\bigcup_{f \in F} T_{f \rightarrow(\Delta)}
$$

where $F=\left\{f: f \in \omega^{\omega \uparrow}, \exists k<\omega, f(i)=i+k \forall i \geqslant k\right\}$. But $F$ is countable. So if $\nu S_{\Delta}>0$ there is an $f \in F$ such that $0<\nu\left(T_{f \rightarrow(\Delta)}\right)=\nu\left(T_{\Delta}\right)$.

If $\nu \in M_{1}\left(\mathscr{P} \omega^{2 \uparrow}\right)$ is deletion-invariant, $w(\nu)=\nu U_{01}=\nu U_{i j}$ whenever $i<j<\omega$.

1E. Proposition. For any $\Delta \subseteq \omega^{2 \uparrow}$ and any $u \in[0,1]$, the following are equivalent:

(i) $(\omega, 2, u) \Rightarrow \Delta$;

(ii) whenever $\nu \in M_{1}\left(\mathscr{P} \omega^{2 \uparrow}\right)$ and $w(\nu) \geqslant u$, then $\nu S_{\Delta}>0$.

Proof. (a) (i) $\Rightarrow$ (ii). If $\Delta=\varnothing$, this is trivial. Otherwise, suppose that $(\omega, 2, u) \Rightarrow$ $\Delta, \nu \in M_{1}\left(\mathscr{P} \omega^{2 \uparrow}\right)$ and $w(\nu) \geqslant u$. As $\nu$ is a Radon probability on a compact metric space, there is a function $\varphi:[0,1] \rightarrow \mathscr{P} \omega^{2 \uparrow}$ which is inverse-measure-preserving for $\mu_{L}$ and $\nu$; that is, $\mu_{L} \varphi^{-1}[E]$ exists and is equal to $\nu E$ whenever $E \subseteq \mathscr{P} \omega^{2 \uparrow}$ is $\nu$-measurable. For $i<j<\omega$ set

Then, if $t \in[0,1]$,

$$
E_{i j}=\varphi^{-1}\left[U_{i j} \backslash S_{\Delta}\right] \subseteq[0,1] .
$$

$$
\Gamma_{t}=\left\{(i, j): t \in E_{i j}\right\}= \begin{cases}\varphi(t) & \text { if } \varphi(t) \notin S_{\Delta} \\ \varnothing & \text { if } \varphi(t) \in S_{\Delta}\end{cases}
$$


So $\Gamma_{t}$ never belongs to $S_{\Delta}$ and $\Delta$ never appears in $\Gamma_{t}$. Consequently, as we are supposing that $(\omega, 2, u) \Rightarrow \Delta$, there are $i<j$ such that $\mu_{L} E_{i j}<u$; so that $\nu\left(U_{i j} \backslash S_{\Delta}\right)<u$ and $\nu S_{\Delta}>0$, as claimed.

(b) (ii) $\Rightarrow$ (i). Suppose that $\nu S_{\Delta}>0$ whenever $\nu \in M_{1}\left(\mathscr{P} \omega^{2 \uparrow}\right)$ and $w(\nu) \geqslant u$, and that $\left\langle E_{i j}\right\rangle_{i<j<\omega}$ is a family of measurable subsets of $[0,1]$ with $\mu_{L} E_{i j} \geqslant u$ for every $i<j$. Define $\varphi:[0,1] \rightarrow \mathscr{P} \omega^{2 \uparrow}$ by writing

$$
\varphi(t)=\left\{(i, j): t \in E_{i j}\right\} \quad \forall t \in[0,1] .
$$

Then $\varphi^{-1}\left[U_{i j}\right]=E_{i j}$ is measurable for every $i<j$. As $\mathscr{P} \omega^{2 \uparrow}$ is separable and metrizable, $\varphi$ is almost continuous, and induces a Radon measure $\nu$ on $\mathscr{P} \omega^{2 \uparrow}$ defined by saying that $\nu E=\mu_{L} \varphi^{-1}[E]$ whenever $E \subseteq \mathscr{P} \omega^{2 \uparrow}$ and $\varphi^{-1}[E]$ is Lebesgue measurable. Now $w(\nu) \geqslant u$, so $\nu S_{\Delta}>0$ and

$$
\{t: \Delta \subseteq \varphi(t)\}=\varphi^{-1}\left[S_{\Delta}\right] \neq \varnothing .
$$

As $\left\langle E_{i j}\right\rangle_{i<j<\omega}$ is arbitrary, $(\omega, 2, u) \Rightarrow \Delta$.

1F. Corollary. Let $\Delta \subseteq \omega^{2 \uparrow}$ and $\nu \in M_{1}\left(\mathscr{P} \omega^{2 \uparrow}\right)$. If $u_{0}(\Delta)<1$, then $\nu\left(S_{\Delta}\right) \geqslant$ $\left(w(\nu)-u_{0}(\Delta)\right) /\left(1-u_{0}(\Delta)\right)$.

Proof. We need only consider the case $\nu S_{\Delta}<1$. In this case, we can define $\nu_{1} \in M_{1}\left(\mathscr{P} \omega^{2 \uparrow}\right)$ by writing

$$
\nu_{1} E=\nu\left(E \backslash S_{\Delta}\right) /\left(1-\nu S_{\Delta}\right)
$$

whenever $E \subseteq \mathscr{P} \omega^{2 \uparrow}$ is such that $E \backslash S_{\Delta}$ is $\nu$-measurable. Then $\nu_{1} S_{\Delta}=0$, so

$$
u_{0}(\Delta) \geqslant w\left(\nu_{1}\right) \geqslant\left(w(\nu)-\nu S_{\Delta}\right) /\left(1-\nu S_{\Delta}\right) .
$$

Rearranging this inequality we obtain the result.

1G. h-representable measures. A particular class of measures on $\mathscr{P} \omega^{2 \uparrow}$ will be of special importance to us, as will appear in $\$ 2$, for reasons described in $\S 5$. Let $(X, \mu)$ be a probability space and $h: X^{2} \rightarrow[0,1]$ a measurable function. Then we have a measure $\lambda_{h}$ on $\mathscr{P} \omega^{2 \uparrow}$ defined by writing

$$
\lambda_{h}\left(T_{\Delta}\right)=\int_{X} \cdots \int_{X} \prod_{(i, j) \in \Delta} h\left(x_{i}, x_{j}\right) d x_{0} \cdots d x_{p}
$$

whenever $p<\omega$ and $\Delta \subseteq(p+1)^{2 \uparrow}$. To see that there is indeed such a measure, it is perhaps easiest to see first that there is an additive functional $\lambda_{h}$ defined on the open-and-closed sets of $\mathscr{P} \omega^{2 \uparrow}$ by writing

$$
\begin{aligned}
\lambda_{h}\left(\left\{\Gamma: \Gamma \subseteq \omega^{2 \uparrow}, \Gamma \cap(p+1)^{2}=\Delta\right\}\right) \\
\quad=\int_{X} \cdots \int_{X} \prod_{(i, j) \in \Delta} h\left(x_{i}, x_{j}\right) \prod_{\substack{i<j \leqslant p \\
(i, j) \notin \Delta}}\left(1-h\left(x_{i}, x_{j}\right)\right) d x_{0} \cdots d x_{p}
\end{aligned}
$$

whenever $p<\omega$ and $\Delta \subseteq(p+1)^{2 \uparrow}$; now because $0 \leqslant h \leqslant 1, \lambda_{h}$ is positive and has a (unique) extension to a Radon measure on $\mathscr{P} \omega^{2 \uparrow}$. Measures representable in this way will be called $h$-representable. Note that such measures are all deletion-invariant, and that $w\left(\lambda_{h}\right)=\iint h(x, y) d x d y$. 
1H. We conclude this section with an elementary lemma.

Lemma. Let $\Delta, H$ be relations. Suppose that $H$ is finite and admits $\Delta^{\prime}$ for every finite $\Delta^{\prime} \subseteq \Delta$. Then $H$ admits $\Delta$.

Proof. For any finite $I \subseteq V(\Delta)$, there is a function $f_{I}: I \rightarrow V(H)$ such that $f_{I}\left(\Delta \cap I^{2}\right) \subseteq H$. Let $\mathscr{F}$ be an ultrafilter on $[V(\Delta)]^{<\omega}$ containing $\{I: J \subseteq I \in$ $\left.[V(\Delta)]^{<\omega}\right\}$ for every $J \in[V(\Delta)]^{<\omega}$. As $H$ is finite,

$$
f(x)=\lim _{I \rightarrow \mathscr{F}} f_{I}(x)
$$

exists for every $x \in V(\Delta)$. $f \rightarrow(\Delta) \subseteq H$, so $H$ admits $\Delta$.

2. The main theorem. The principal result of this paper is Theorem $2 \mathrm{~J}$, which shows how to reduce the calculation of $u_{0}(\Delta)$ to a problem in finite combinatorics. We approach this through Theorem $2 \mathrm{~F}$, which gives a sufficient condition for $\Delta$ to appear in $\Gamma$. Our results depend upon a new method of applying the Radon-Nikodým theorem to repeated limits.

2A. Measures on $\beta \omega$. While the results below are important to us because they refer to certain additive functionals on $\mathscr{P} \omega^{2}$, we find them very much easier to approach if they are expressed in terms of Radon measures on $\omega^{*}=\beta \omega \backslash \omega$, the Stone-Čech remainder of $\omega$. Recall that $\omega^{*}$ is a zero-dimensional compact Hausdorff space, and its algebra of open and closed sets is $\left\{A^{*}: A \subseteq \omega\right\}$, where $A^{*}=\{\mathscr{F}$ : $\left.A \in \mathscr{F} \in \omega^{*}\right\}$ for each $A \subseteq \omega$; the map $A \mapsto A^{*}$ induces an isomorphism between $\mathscr{P} \omega /[\omega]^{<\omega}$ and the algebra of open and closed sets in $\omega^{*}$. There is accordingly a natural correspondence between Radon measures $\mu$ on $\omega^{*}$ and nonnegative additive functionals $\vartheta: \mathscr{P} \omega \rightarrow \mathbf{R}$ which are zero on $[\omega]^{<\omega}$, given by writing $\vartheta A=\mu A^{*}$ for $A \subseteq \omega$.

At the same time, if $f \in \ell^{\infty}(\omega)$, we have a function $f^{*}: \omega^{*} \rightarrow \mathbf{R}$ given by $f^{*}(\mathscr{F})=\lim _{n \rightarrow \mathscr{F}} f(n)$ for $\mathscr{F} \in \omega^{*}$; the map $f \mapsto f^{*}$ induces a Banach lattice isomorphism between $\ell^{\infty}(\omega) / c_{0}(\omega)$ and $C\left(\omega^{*}\right)$. We therefore have a natural correspondence between Radon measures $\mu$ on $\omega^{*}$ and nonnegative linear functionals $\varphi$ on $\ell^{\infty}(\omega)$ which vanish on $c_{0}(\omega)$, given by writing $\varphi(f)=\int f^{*} d \mu$.

We shall repeatedly use the following elementary fact: if $\mu$ is a Radon measure on $\omega^{*}, E \subseteq \omega^{*}$ is $\mu$-measurable, and $\varepsilon>0$, there is an $A \subseteq \omega$ such that $\mu\left(E \Delta A^{*}\right) \leqslant \varepsilon$; this is because any two disjoint compact subsets of $\omega^{*}$ can be separated by an open-and-closed set.

2B. The fundamental construction. Let $\mu$ be a Radon measure on $\omega^{*}$ and $\Gamma$ any subset of $\omega^{2}$. For $a<\omega$ write

$$
\begin{gathered}
\Gamma[\{a\}]=\{b:(a, b) \in \Gamma\}, \\
f_{\Gamma}(a)=\mu\left(\Gamma[\{a\}]^{*}\right), \quad \zeta(\Gamma, \mu)=\int f_{\Gamma}^{*} d \mu,
\end{gathered}
$$

where $A^{*}$ and $f_{\Gamma}^{*}$ carry the meanings assigned in $2 \mathrm{~A}$. Then we see that, for fixed $\mu$, $\Gamma \mapsto f_{\Gamma}$ and $\Gamma \mapsto \zeta(\Gamma, \mu)$ are nonnegative additive functions on $\mathscr{P} \omega^{2}$.

Now write $\Sigma$ for the domain of $\mu$, and keep $\Gamma$ fixed for a paragraph. For $E$, $F \in \Sigma$, write

$$
g_{F}(a)=\mu\left(F \cap \Gamma[\{a\}]^{*}\right) \quad \forall a<\omega, \quad \varphi(E, F)=\int_{E} g_{F}^{*} d \mu .
$$


Then we see that $\varphi$ is biadditive (i.e. the maps $E \mapsto \varphi(E, F)$ and $E \mapsto \varphi(F, E)$ are additive for fixed $F \in \Sigma)$, and that $0 \leqslant \varphi(E, F) \leqslant \mu E . \mu F=\mu^{2}(E \times F)$ for all $E$, $F \in \Sigma$, where $\mu^{2}$ is the product measure on $\omega^{* 2}$. There is therefore a unique additive functional $\psi: \Sigma \otimes \Sigma \rightarrow \mathbf{R}$ such that $\psi(E \times F)=\varphi(E, F)$ for all $E, F \in \Sigma$, where $\Sigma \otimes \Sigma$ is the algebra of subsets of $\omega^{*} \times \omega^{*}$ generated by $\{E \times F: E, F \in \Sigma\}$; and we still have $0 \leqslant \psi(G) \leqslant \mu^{2}(G)$ for every $G \in \Sigma \otimes \Sigma$. Next, it follows from this that $\psi$ has a unique extension to the domain of $\mu^{2}$ which is still additive and dominated by $\mu^{2}$. By the Radon-Nikodým theorem (this is the key idea of this paper), there is a $\mu^{2}$-measurable function $h: \omega^{*} \times \omega^{*} \rightarrow[0,1]$ such that $\psi(G)=\int_{G} h d \mu^{2}$ for every $\mu^{2}$-measurable $G$. Of course we can suppose (as will be convenient later) that $h$ is measurable for the $\sigma$-algebra $\Sigma \hat{\otimes}_{\sigma} \Sigma$ generated by $\Sigma \otimes \Sigma$.

Working back through the definitions, we see that $h$ has the property that

$$
\iint_{A^{*} \times B^{*}} h(x, y) d x d y=\int g_{A B}^{*} d \mu \quad \forall A, B \subseteq \omega
$$

where

$$
g_{A B}(a)= \begin{cases}\mu(B \cap \Gamma[\{a\}])^{*} & \text { if } a \in A \\ 0 & \text { if } a \in \omega \backslash A\end{cases}
$$

We see also that

$$
\iint h(x, y) d x d y=\zeta(\Gamma, \mu)
$$

2C. Definition. For any $\Gamma \subseteq \omega^{2}$, write $\zeta_{0}(\Gamma)=\sup \{\zeta(\Gamma, \mu): \mu$ is a Radon probability on $\left.\omega^{*}\right\}$. Observe that $\zeta_{0}$ is a subadditive functional, and that $\zeta_{0}(\Gamma)=$ $\zeta_{0}\left(\Gamma \cap \omega^{2 \uparrow}\right)$ for every $\Gamma$ (because $\zeta\left(\omega^{2} \backslash \omega^{2 \uparrow}, \mu\right)=0$ for every $\left.\mu\right)$.

2D. LemmA. Let $\Gamma, \mu$ and $h$ be related as in 2B. Suppose that $\left\langle E_{r}\right\rangle_{r<p}$ is a disjoint finite sequence of $\mu$-measurable sets, where $p \geqslant 1$, and that $K \subseteq p^{2}$ is such that $(r, r) \notin K$ for all $r<p$ and

$$
\mu^{p}\left\{\mathbf{x}: \mathbf{x} \in \prod_{r<p} E_{r}, h\left(x_{r}, x_{s}\right)>0 \forall(r, s) \in K\right\}>0,
$$

where $\mu^{p}$ is the product measure on $\left(\omega^{*}\right)^{p}$. Let $\left\langle r_{k}\right\rangle_{k<\omega} \in p^{\omega}$. Then there is a strictly increasing sequence $\left\langle i_{k}\right\rangle_{k<\omega}$ such that $\left(i_{k}, i_{l}\right) \in \Gamma$ whenever $k<l$ and $\left(r_{k}, r_{l}\right) \in K$.

Proof. Let $\alpha>0$ be such that

$$
\mu^{p}\left\{\mathbf{x}: \mathbf{x} \in \prod_{r<p} E_{r}, h\left(x_{r}, x_{s}\right) \geqslant \alpha \forall(r, s) \in K\right\}>0 .
$$

Set $H=\{(x, y): h(x, y) \geqslant \alpha\} \subseteq \omega^{* 2}$,

$$
\begin{aligned}
G & =\left\{\mathbf{x}: \mathbf{x} \in \omega^{* p}, h\left(x_{r}, x_{s}\right) \geqslant \alpha \forall(r, s) \in K\right\}, \\
\eta & =\alpha^{2} /\left(4 p^{2}+1\right)>0 .
\end{aligned}
$$


We seek to define, by induction, sequences $\left\langle i_{l}\right\rangle_{l<\omega}$ in $\omega$ and $\left\langle A_{l r}\right\rangle_{l<\omega, r<p}$ in $\mathscr{P} \omega$, with the following properties:

$$
\begin{gathered}
\mu^{p}\left(G \cap \prod_{r<p} A_{l r}^{*}\right)>0 ; \\
A_{l r} \cap A_{l s}=\varnothing \text { if } r<s<p ; \\
A_{l r} \subseteq \Gamma\left[\left\{i_{k}\right\}\right] \text { whenever } k<l \text { and }\left(r_{k}, r\right) \in K ; \\
i_{k}<i_{l} \text { whenever } k<l ; \\
\left(i_{k}, i_{l}\right) \in \Gamma \text { whenever } k<l \text { and }\left(r_{k}, r_{l}\right) \in K .
\end{gathered}
$$

We start the induction by observing that, as $\omega^{*}$ is zero-dimensional and every open-and-closed subset is of the form $A^{*}$, we can find $\left\langle A_{0 r}\right\rangle_{r<p}$ such that $\mu\left(E_{r} \Delta A_{0 r}^{*}\right)$ is as small as we please for each $r$, and in particular such that $\mu^{p}\left(G \cap \Pi_{r<p} A_{0 r}^{*}\right)>0$; and with a little more care we can arrange that $A_{0 r} \cap A_{0 s}=\varnothing$ if $r<s<p$. For the inductive step to $l+1$, start by taking measurable sets $F_{r} \subseteq A_{l r}^{*}$ such that $\mu F_{r}>0$ for $r<p$ and

$$
\mu^{p}\left(G \cap \prod_{r<p} F_{r}\right)>(1-\eta) \prod_{r<p} \mu F_{r} .
$$

Let $B_{r} \subseteq A_{l r}$ be such that $\mu\left(B_{r}^{*} \Delta F_{r}\right)$ is sufficiently small so that

$$
\mu^{p}\left(G \cap \prod_{r<p} B_{r}^{*}\right) \geqslant(1-\eta) \prod_{r<p} \mu B_{r}^{*}>0 .
$$

Then for each $(r, s) \in K$,

$$
\mu^{2}\left(\left(B_{r}^{*} \times B_{s}^{*}\right) \backslash H\right) \leqslant \eta \mu B_{r}^{*} \mu B_{s}^{*} .
$$

Set $I=\left\{s:\left(r_{l}, s\right) \in K\right\}$, and for $s \in I$ set

$$
D_{s}=\left\{j: j \in B_{r_{l}}, \mu\left(B_{s} \cap \Gamma[\{j\}]\right)^{*} \leqslant(\alpha / 2) \mu B_{s}^{*}\right\} .
$$

Then we see that

$$
\int_{D_{s}^{*} \times B_{s}^{*}} h \leqslant \frac{\alpha}{2} \mu D_{s}^{*} \mu B_{s}^{*}
$$

so that

$$
\eta \mu B_{r_{l}}^{*} \mu B_{s}^{*} \geqslant \mu^{2}\left(\left(B_{r_{l}}^{*} \times B_{s}^{*}\right) \backslash H\right) \geqslant \mu^{2}\left(\left(D_{s}^{*} \times B_{s}^{*}\right) \backslash H\right) \geqslant \frac{1}{2} \mu D_{s}^{*} \mu B_{s}^{*},
$$

and

$$
\mu D_{s}^{*} \leqslant 2 \eta \mu B_{r_{l}}^{*} \quad \forall s \in I .
$$

As $2 \eta p<1, \mu\left(B_{r_{l}}^{*} \backslash \bigcup_{s \in I} D_{s}^{*}\right)>0$ and $B_{r_{l}} \backslash \bigcup_{s \in I} D_{s}$ is infinite; let $i_{l} \in B_{r_{l}} \backslash \bigcup_{s \in I} D_{s}$ be such that $i_{k}<i_{\text {l }}$ whenever $k<l$. We have $\mu\left(B_{s} \cap \Gamma\left[\left\{i_{l}\right\}\right]\right)^{*}>\frac{1}{2} \alpha \mu B_{s}^{*}$ for every $s \in I$. Because $i_{l} \in B_{r_{l}} \subseteq A_{l r_{l}}$, we have $i_{l} \in \Gamma\left[\left\{i_{k}\right\}\right]$ when $k<l$ and $\left(r_{k}, r_{l}\right) \in K$, i.e. $\left(i_{k}, i_{l}\right) \in \Gamma$ whenever $k<l$ and $\left(r_{k}, r_{l}\right) \in K$.

Try

$$
A_{l+1, r}= \begin{cases}B_{r} \cap \Gamma\left[\left\{i_{l}\right\}\right] & \text { for } r \in I, \\ B_{r} & \text { for } r \in p \backslash I .\end{cases}
$$


Then $A_{l+1, r} \subseteq A_{l r} \subseteq \Gamma\left[\left\{i_{k}\right\}\right]$ whenever $k<l$ and $\left(r_{k}, r\right) \in K$; at the same time, $A_{l+1, r} \subseteq \Gamma\left[\left\{i_{l}\right\}\right]$ whenever $\left(r_{l}, r\right) \in K$. Thus we have achieved $(\beta),(\gamma),(\delta)$ and $(\varepsilon)$ at level $l+1$. We still have to check $(\alpha)$ at this level. But if $(r, s) \in K$,

$$
\mu^{2}\left(\left(A_{l+1, r}^{*} \times A_{l+1, s}^{*}\right) \backslash H\right) \leqslant \eta \mu B_{r}^{*} \mu B_{s}^{*} \leqslant \eta(2 / \alpha)^{2} \mu A_{l+1, r}^{*} \mu A_{l+1, s}^{*} .
$$

So

$$
\begin{aligned}
\mu^{p}\left(\prod_{r<p} A_{l+1, r}^{*} \backslash G\right) & \leqslant \#(K) \eta\left(\frac{2}{\alpha}\right)^{2} \prod_{r<p} \mu A_{l+1, r}^{*} \\
& <\prod_{r<p} \mu A_{l+1, r}^{*},
\end{aligned}
$$

and $\mu^{p}\left(\prod_{r<p} A_{l+1, r}^{*} \cap G\right)>0$. Thus the induction continues.

2E. Lemma. Let $\Gamma \subseteq \omega^{2 \uparrow}$ and let $\mu$ be a Radon probability on $\omega^{*}$. Construct $h: \omega^{* 2} \rightarrow[0,1]$ as in $2 \mathrm{~B}$. Suppose that there is a measurable $E \subseteq \omega^{*}$ such that $\mu E>0$ and $\int_{F \times F} h>0$ whenever $F \subseteq E$ is measurable and $\mu F>0$. Then $\omega^{2 \uparrow} \subseteq \Gamma$.

Proof. (a) We show first that if $A \subseteq \omega$ and $\mu\left(E \cap A^{*}\right)>0$, there is an $a \in A$ such that $\mu\left(E \cap A^{*} \cap \Gamma[\{a\}]^{*}\right)>0$. For set $F=E \cap A^{*}$ and let $\delta$ be such that $\int_{F \times F} h=3 \delta(\mu F)^{2}$. Then there is a $B \subseteq A$ such that

$$
\int_{B^{*} \times B^{*}} h \geqslant 2 \delta\left(\mu B^{*}\right)^{2}, \quad \mu\left(B^{*} \triangle F\right) \leqslant \delta \mu B^{*} .
$$

Set

$$
\begin{aligned}
g(a) & =\mu(B \cap \Gamma[\{a\}])^{*} & & \text { if } a \in B, \\
& =0 & & \text { if } a \in \omega \backslash B ;
\end{aligned}
$$

then $\int g^{*} d \mu=\int_{B^{*} \times B^{*}} h$. Since $g^{*}$ is zero off $B^{*}$, there is an $\mathscr{F} \in B^{*}$ such that $g^{*}(\mathscr{F}) \geqslant 2 \delta \mu B^{*}$, and there is an $a \in B$ such that $g(a)>\delta \mu B^{*}$; so that $\mu(B \cap \Gamma[\{a\}])^{*}>\delta \mu B^{*}$ and $\mu\left(B^{*} \cap F \cap \Gamma[\{a\}]^{*}\right)>0$, as required.

(b) Now we can choose $\left\langle a_{k}\right\rangle_{k<\omega}$ inductively so that

$$
a_{k} \in A_{k}=\omega \cap \bigcap_{i<k} \Gamma\left[\left\{a_{i}\right\}\right], \quad \mu\left(E \cap A_{k}^{*}\right)>0
$$

for every $k<\omega$; in this case $\left(a_{i}, a_{k}\right) \in \Gamma$ whenever $i<k$ so that $\omega^{2 \uparrow} \subset \Gamma$.

2F. ThEOREM. Let $\Gamma, \Delta \subseteq \omega^{2 \uparrow}$. Set

$$
u_{3}=\sup \left\{p^{-2} \#(H): p \geqslant 1, H \subseteq p^{2}, H \text { does not admit } \Delta\right\} .
$$

If $\zeta_{0}(\Gamma)>u_{3}$, then $\Delta \subseteq \Gamma$.

REMARK. In $2 \mathrm{~J}$ below we shall show that $u_{3}=u_{0}(\Delta)$.

Proof of Theorem 2F. (a) Let $\mu$ be a Radon probability on $\omega^{*}$ such that $\zeta(\Gamma, \mu)>u_{3}$. Let $\Sigma$ be the domain of $\mu$ and construct a $\Sigma \hat{\otimes}_{\sigma} \Sigma$-measurable function $h: \omega^{* 2} \rightarrow[0,1]$ as in $2 \mathrm{~B}$ above. Set $H=\{(x, y): h(x, y)>0\}$; then

$$
\mu^{2} H \geqslant \int h=\zeta(\Gamma, \mu)>u_{3} .
$$

Take $\eta=\left(\mu^{2} H-u_{3}\right) / 3>0, p \geqslant 2$ such that $p \eta>1$, and $\varepsilon=1 /\left(p^{2}+1\right)>0$. 
(b) We have to dispose of the possibility that there is an atom $E$ for $\mu$ such that $\mu^{2}((E \times E) \cap H)>0$. But if there is such a set, it satisfies the condition of Lemma $2 \mathrm{E}$, and $\omega^{2 \uparrow} \subseteq \Gamma$; a fortiori, $\Delta \subseteq \Gamma$. We shall therefore assume from now on that $\mu^{2}((E \times E) \cap H)=0$ for every atom $E \subseteq \omega^{*}$.

(c) For each finite dissection $\mathscr{E}$ of $\omega^{*}$ into measurable sets, let $f_{\mathscr{E}}$ be the corresponding approximation to $\chi H$, given by

$$
f_{\mathscr{E}}(x, y)=\frac{1}{\mu E \cdot \mu F} \mu(H \cap(E \times F)) \text { whenever } x \in E \in \mathscr{E}, y \in F \in \mathscr{E} .
$$

Then we can find an $\mathscr{E}$ such that

$$
\int\left|\chi H-f_{\mathscr{E}}\right| \leqslant \varepsilon \eta
$$
every element of $\mathscr{E}$ is either an atom or has measure $\leqslant \eta / p^{2}$.

Set $H^{\prime}=\left\{(x, y): f_{\mathscr{E}}(x, y) \geqslant 1-\varepsilon\right\}$. Then $\mu^{2}\left(H \Delta H^{\prime}\right) \leqslant \eta$; also, if $E \in \mathscr{E}$ is an atom, $H^{\prime} \cap(E \times E)=\varnothing$ so that

(d) For each $\mathbf{x} \in \omega^{* p}$ set

$$
\mu^{2}\left(H^{\prime} \cap \bigcup_{E \in \mathscr{E}}(E \times E)\right) \leqslant \eta / p^{2} .
$$

$$
K_{\mathrm{x}}=\left\{(r, s):\left(x_{r}, x_{s}\right) \in H^{\prime}\right\} \subseteq p^{2} .
$$

Then

$$
\int \#\left(K_{\mathbf{x}}\right) d \mathbf{x}=p^{2} \mu^{2} H^{\prime}
$$

At the same time, writing

$$
G=\left\{\mathbf{x}: \mathbf{x} \in \omega^{* p}, \exists r<s<p,\left(x_{r}, x_{s}\right) \in H^{\prime} \cap \bigcup_{E \in \mathscr{E}}(E \times E)\right\},
$$

$\mu^{p} G \leqslant p(p-1) \eta / 2 p^{2} \leqslant \eta$. So

$$
\int_{\omega^{* p} \backslash G} \#\left(K_{\mathbf{x}}\right) d \mathbf{x} \geqslant p^{2}\left(\mu^{2} H^{\prime}-\eta\right),
$$

and there is an $\mathbf{x} \in \omega^{* p} \backslash G$ such that $\#\left(K_{\mathbf{x}}\right) \geqslant p^{2}\left(\mu^{2} H^{\prime}-\eta\right)$. Set

$$
K_{\mathbf{x}}^{\prime}=K_{\mathbf{x}} \backslash\{(r, r): r<p\} ;
$$

then $\#\left(K_{\mathbf{x}}^{\prime}\right) \geqslant p^{2}\left(\mu^{2} H-2 \eta\right)-p>p^{2} u_{3}$.

(e) Let $\left\langle E_{r}\right\rangle_{r<q}$, where $q \leqslant p$, enumerate

$$
\left\{E: \exists r<p, x_{r} \in E \in \mathscr{E}\right\} \text {. }
$$

As $\#\left(K_{\mathbf{x}}^{\prime}\right)>p^{2} u_{3}, K_{\mathbf{x}}^{\prime}$ admits $\Delta$; let $\left\langle r_{i}\right\rangle_{i<\omega} \in p^{\omega}$ be such that $\left(r_{i}, r_{j}\right) \in K_{\mathbf{x}}^{\prime}$ whenever $(i, j) \in \Delta$, and define $s_{i}<q$ by saying that $x_{r_{i}} \in E_{s_{i}}$ for each $i<\omega$. Consider

$$
K=\left\{\left(s_{i}, s_{j}\right):(i, j) \in \Delta\right\} \subseteq q^{2} .
$$

If $(i, j) \in \Delta$, then $\left(r_{i}, r_{j}\right) \in K_{\mathbf{x}}^{\prime}$, so $r_{i} \neq r_{j}$; consequently, as $\left(x_{r_{i}}, x_{r_{j}}\right) \in H^{\prime}$ and $\mathbf{x} \notin G, s_{i} \neq s_{j}$; thus $(s, s) \notin K$ for $s<q$. Also, if $(i, j) \in \Delta$, then $\left(x_{r_{i}}, x_{r_{i}}\right) \in H^{\prime}$, so $E_{s_{i}} \times E_{s,} \subseteq H^{\prime}$, and

(f) Thus

$$
\mu^{2}\left(H \cap\left(E_{s_{i}} \times E_{s_{j}}\right)\right) \geqslant(1-\varepsilon) \mu E_{s_{i}} \mu E_{s_{j}} .
$$

$$
\mu^{2}\left(E_{r} \times E_{s}\right) \backslash H \leqslant \varepsilon \mu E_{r} \mu E_{s} \quad \forall(r, s) \in K
$$


It follows that

$$
\mu^{q}\left\{\mathbf{y}: \mathbf{y} \in \prod_{r<q} E_{r}, \exists(r, s) \in K,\left(y_{r}, y_{s}\right) \notin H\right\} \leqslant q^{2} \varepsilon \prod_{r<q} \mu E_{r},
$$

and

$$
\mu^{q}\left\{\mathbf{y}: \mathbf{y} \in \prod_{r<q} E_{r},\left(y_{r}, y_{s}\right) \in H \forall(r, s) \in K\right\}>0 .
$$

Since $\left(s_{i}, s_{j}\right) \in K$ whenever $(i, j) \in \Delta$, this enables us to apply Lemma 2D, and we conclude that $\Delta \subseteq \Gamma$, as stated.

To relate this result to our original problems we need the following elementary lemma.

2G. LemmA. Let $H$ be a Hilbert space and $\left\langle x_{i j}\right\rangle_{i \leqslant n, j<\omega}$ a bounded double sequence in $H$, where $n<\omega$. Suppose that $\mathscr{F}$ is a nonprincipal ultrafilter on $\omega$ and that $x_{i}$ is the weak limit along $\mathscr{F}$ of $\left\langle x_{i j}\right\rangle_{j<\omega}$ for each $i \leqslant n$. Then for any $\varepsilon>0, r<\omega$ there is $a$ finite $J \subseteq \omega$ such that $\#(J) \geqslant r$ and

$$
\left\|\frac{1}{\#(J)} \sum_{j \in J} x_{i j}-x_{i}\right\| \leqslant \varepsilon \quad \forall i \leqslant n .
$$

Proof. (a) $n=0$. If $H$ is finite dimensional the result is elementary; we suppose that $H$ is infinite dimensional. Clearly it is enough to consider the case in which $x_{0}=0$ and $\left\|x_{0 j}\right\| \leqslant 1$ for each $j<\omega$. Take $m \geqslant r$ so large that $m \varepsilon^{2} \geqslant 4$, and define $r_{0}<r_{1}<\cdots$ in $\omega, e_{0}, e_{1}, \ldots \in H$ inductively, as follows.

$r_{0}=0$. Given $r_{k}$ and $e_{l}$ for $l \leqslant k$, let $e_{k}$ be such that $\left\|e_{k}\right\|=1,\left(e_{l} \mid e_{k}\right)=0$ for $l<k$, and $x_{0 r_{k}} \in \operatorname{lin}\left\{e_{0}, \ldots, e_{k}\right\}$; let $r_{k+1}$ be such that $\left(x_{0 r_{k+1}} \mid e_{l}\right) \leqslant 2^{-k-1}$ for $l \leqslant k$. We continue until we have $r_{m}$ and $e_{m}$.

Now consider $z=\frac{1}{m+1} \sum_{k \leqslant m} x_{0 r_{k}}$. As $x_{0 r_{k}} \in \operatorname{lin}\left\{e_{0}, \ldots, e_{m}\right\}$ for any $k \leqslant m$,

$$
\begin{aligned}
\|z\|^{2} & =\frac{1}{(m+1)^{2}} \sum_{l \leqslant m}\left|\sum_{k \leqslant m}\left(x_{r_{k}} \mid e_{l}\right)\right|^{2} \\
& \leqslant \frac{1}{(m+1)^{2}} \sum_{l \leqslant m}\left|1+\sum_{l<k \leqslant m} 2^{-k}\right|^{2} \leqslant \frac{4}{m+1} \leqslant \varepsilon^{2} .
\end{aligned}
$$

So $\|z\| \leqslant \varepsilon$. Thus $J=\left\{r_{k}: k \leqslant m\right\}$ will serve.

(b) Now the general case follows by working in the Hilbert space $G=H \oplus H \oplus$ $\cdots \oplus H$, the Hilbert space sum of $n+1$ copies of $H$, and identifying $\left\langle x_{i j}\right\rangle_{i \leqslant n}$ with an element of $G$ for each $j<\omega$.

2H. Lemma. Let $\nu \in M_{1}\left(\mathscr{P} \omega^{2 \uparrow}\right)$. Then there is a sequence $\left\langle I_{n}\right\rangle_{n<\omega}$ of nonempty finite subsets of $\omega$ such that

(i) $\max I_{n}<\min I_{n+1} \forall n<\omega$;

(ii) for every $p<\omega$ and $\Delta \subseteq(p+1)^{2 \uparrow}$,

$$
\lim _{n_{0} \rightarrow \infty} \cdots \lim _{n_{p} \rightarrow \infty} \frac{\#\left\{\sigma: \sigma \in \prod_{i \leqslant p} I_{n_{i}}, \sigma \rightarrow(\Delta) \subseteq \Gamma\right\}}{\#\left(\prod_{i \leqslant p} I_{n_{i}}\right)}
$$

exists for $\nu$-almost all $\Gamma \subseteq \omega^{2 \uparrow}$. 
REMARK. For the principal results of this paper we need only the case $p=1$, $\Delta=\{(0,1)\}$; the general formula above will not be used except in $\S 5$.

Proof. Let $\mathscr{F}$ be a nonprincipal ultrafilter on $\omega$. For $\Delta \subseteq(p+1)^{2 \uparrow}, \sigma \in \bigcup_{k<\omega} \omega^{k}$ define $\nu$-measurable functions $g_{\sigma}^{\Delta}: \mathscr{P} \omega^{2 \uparrow} \rightarrow[0,1]$ as follows. If $\sigma \in \omega^{k}$ where $k \geqslant$ $p+1$, say

$$
g_{\sigma}^{\Delta}(\Gamma)= \begin{cases}1 & \text { if } \sigma \rightarrow(\Delta) \subseteq \Gamma, \\ 0 & \text { otherwise. }\end{cases}
$$

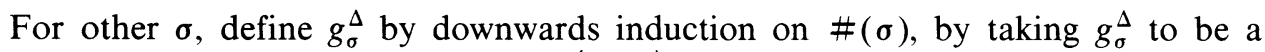
$\mathfrak{I}_{s}\left(\mathscr{L}^{2}(\nu), \mathscr{L}^{2}(\nu)\right)$-limit along $\mathscr{F}$ of $\left\langle g_{\sigma \frown i}^{\Delta}\right\rangle_{i<\omega}$, where $\sigma \frown i$ is that extension of $\sigma$ such that

$$
\operatorname{dom}(\sigma \frown i)=\operatorname{dom}(\sigma)+1 \text { and }(\sigma \frown i)(\operatorname{dom}(\sigma))=i .
$$

Thus $\int_{E} g_{\sigma}^{\Delta} d \nu=\lim _{i \rightarrow \mathscr{F}} \int_{E} g_{\sigma \frown i}^{\Delta} d \nu$ for every $\nu$-measurable set $E$. (Note that of course this last formula is valid when $\operatorname{dom}(\sigma) \geqslant p+1$, as then $g_{\sigma}^{\Delta} \wedge_{i}=g_{\sigma}^{\Delta}$ for every $i<\omega$.)

Now we choose $\left\langle I_{n}\right\rangle_{n<\omega}$ to be a sequence of nonempty finite subsets of $\omega$ such that

$$
\begin{gathered}
I_{0}=\{0\} ; \quad \min I_{n+1} \geqslant \max I_{n} ; \\
\left\|\frac{1}{\#\left(I_{n+1}\right)} \sum_{i \in I_{n+1}} g_{\sigma \frown i}^{\Delta}-g_{\sigma}^{\Delta}\right\|_{2} \leqslant 2^{-n} \quad \forall \Delta \subseteq(n+1)^{2 \uparrow}, \sigma \in \bigcup_{k \leqslant n}\left(\max I_{n}\right)^{k},
\end{gathered}
$$

using Lemma $2 \mathrm{G}$. In this case we see that

$$
\lim _{n \rightarrow \infty} \frac{1}{\#\left(I_{n}\right)} \sum_{i \in I_{n}} g_{\sigma{ }_{i}}^{\Delta}(\Gamma)=g_{\sigma}^{\Delta}(\Gamma)
$$

for $\nu$-almost all $\Gamma$, for every $\sigma \in \bigcup_{k<\omega} \omega^{k}$ and finite $\Delta \subseteq \omega^{2 \uparrow}$. Consequently

$$
E=\left\{\Gamma: g_{\sigma}^{\Delta}(\Gamma)=\lim _{n \rightarrow \infty} \frac{1}{\#\left(I_{n}\right)} \sum_{i \in I_{n}} g_{\sigma \frown i}^{\Delta}(\Gamma) \text { for all finite } \Delta \subseteq \omega^{2 \uparrow}, \sigma \in \bigcup_{k<\omega} \omega^{k}\right\}
$$

has measure 1. But if $\Gamma \in E$, the required limit of (ii) exists and is equal to $g_{\varnothing}^{\Delta}(\Gamma)$, for every finite $\Delta$.

2I. Lemma. Suppose that $\nu \in M_{1}\left(\mathscr{P} \omega^{2 \uparrow}\right)$. Then there is a Radon probability $\mu$ on $\omega^{*}$ such that

exists and is greater than 0.

$$
\nu\left\{\Gamma: \Gamma \subseteq \omega^{2 \uparrow}, \zeta(\Gamma, \mu) \geqslant w(\nu)\right\}
$$

Proof. Let $\left\langle I_{n}\right\rangle_{n<\omega}$ be chosen as in $2 \mathrm{H}$. Then, in particular,

$$
g(\Gamma)=\lim _{m \rightarrow \infty} \lim _{n \rightarrow \infty} \frac{\#\left(\Gamma \cap\left(I_{m} \times I_{n}\right)\right)}{\#\left(I_{m} \times I_{n}\right)}
$$

exists for almost all $\Gamma$. Now, by Lebesgue's theorem,

$$
\int g d \nu=\lim _{m \rightarrow \infty} \lim _{n \rightarrow \infty} \frac{1}{\#\left(I_{m} \times I_{n}\right)} \sum_{\substack{a \in I_{m} \\ b \in I_{n}}} \nu U_{a b} \geqslant w(\nu) .
$$

So $\nu\{\Gamma: g(\Gamma) \geqslant w(\nu)\}>0$. 
Now take any nonprincipal ultrafilter $\mathscr{F}$ on $\omega$ and let $\mu$ be the Radon measure on $\omega^{*}$ defined by writing

$$
\mu A^{*}=\lim _{n \rightarrow \mathscr{F}} \frac{\#\left(A \cap I_{n}\right)}{\#\left(I_{n}\right)}
$$

for every $A \subseteq \omega$. (This is well defined because $\lim _{n \rightarrow \infty} \min \left(I_{n}\right)=\infty$.) Let $\Gamma \subseteq \omega^{2 \uparrow}$ be such that $g(\Gamma)$ exists. Then we can calculate $\zeta(\Gamma, \mu)$, as follows. For $a<\omega$, set

$$
f(a)=\mu \Gamma[\{a\}]^{*}=\lim _{n \rightarrow \mathscr{F}} \frac{\#\left(I_{n} \cap \Gamma[\{a\}]\right)}{\#\left(I_{n}\right)} .
$$

Then

$$
\begin{aligned}
\zeta(\Gamma, \mu) & =\int f^{*} d \mu=\lim _{m \rightarrow \mathscr{F}} \frac{1}{\#\left(I_{m}\right)} \sum_{a \in I_{m}} f(a) \\
& =\lim _{m \rightarrow \mathscr{F}} \lim _{n \rightarrow \mathscr{F}} \frac{1}{\#\left(I_{m}\right) \#\left(I_{n}\right)} \sum_{a \in I_{m}} \#\left(I_{n} \cap \Gamma[\{a\}]\right) \\
& =\lim _{m \rightarrow \mathscr{F}} \lim _{n \rightarrow \mathscr{F}} \frac{\#\left(\Gamma \cap\left(I_{m} \times I_{n}\right)\right)}{\#\left(I_{m} \times I_{n}\right)}=g(\Gamma) .
\end{aligned}
$$

So

$$
\nu\{\Gamma: \zeta(\Gamma, \mu) \geqslant w(\nu)\}=\nu\{\Gamma: g(\Gamma) \geqslant w(\nu)\}>0
$$

2J. THEOREM. Let $\Delta \subseteq \omega^{2 \uparrow}$ be nonempty. Then

$$
\begin{aligned}
& u_{1}(\Delta)=\sup \left\{w(\nu): \nu \in M_{1}\left(\mathscr{P} \omega^{2 \uparrow}\right), \nu\left(S_{\Delta}\right)=0\right\}, \\
& u_{2}(\Delta)=\sup \left\{w(\nu): \nu \in M_{1}\left(\mathscr{P} \omega^{2 \uparrow}\right), \nu\left(S_{\Delta}^{*}\right)=0\right\}, \\
& u_{3}(\Delta)=\sup \left\{(H) / p^{2}: p \geqslant 1, H \subseteq p^{2}, H \text { does not admit } \Delta\right\}, \\
& u_{4}(\Delta)=\sup \{v(H): H \text { is a finite relation not admitting } \Delta\}, \\
& u_{5}(\Delta)=\sup \left\{\int h: \text { there is a probability space } X,\right. \\
& \left.\qquad h: X^{2} \rightarrow[0,1] \text { is measurable, and } \lambda_{h}\left(S_{\Delta}\right)=0\right\}, \\
& u_{6}(\Delta)=\sup \left\{\zeta_{0}(\Gamma): \Gamma \subseteq \omega^{2 \uparrow}, \Delta \nsubseteq \Gamma\right\}, \text { and } \\
& u_{7}(\Delta)=\sup _{p<\omega} u_{0}\left(\Delta \cap p^{2}\right),
\end{aligned}
$$

are all equal to $u_{0}(\Delta)$.

Proof. (a) $u_{0}=u_{1}$ by Proposition $1 \mathrm{E}$.

(b) $u_{1} \geqslant u_{5}$. Elementary, as each $\lambda_{h} \in M_{1}\left(\mathscr{P} \omega^{2 \uparrow}\right)$.

(c) $u_{5} \geqslant u_{4}$. Suppose that $H$ is a finite relation not admitting $\Delta$. Then there is a $p<\omega$ such that $H$ does not admit $\Delta \cap p^{2}=\Delta^{\prime}$ (Lemma 1H). If $H=\varnothing$ then of 
course $v(H) \leqslant u_{5}$. Otherwise, there is a probability measure $\mu$ on $V(H)$ such that $\mu^{2}(H)=v(H)$. Consider

$$
\lambda_{\chi H}\left(T_{\Delta^{\prime}}\right)=\sum_{\substack{f \in V(H)^{p} \\ f \rightarrow\left(\Delta^{\prime}\right) \subseteq H}} \prod_{i<p} \mu\{f(i)\}=0 .
$$

As $\lambda_{\chi H^{H}}$ is deletion-invariant, $\lambda_{\chi H}\left(S_{\Delta^{\prime}}\right)=0$ (1D). So $\lambda_{\chi H}\left(S_{\Delta}\right)=0$, and $v(H) \leqslant u_{5}$. As $H$ is arbitrary, $u_{4} \leqslant u_{5}$.

(d) $u_{4} \geqslant u_{3}$. Elementary, as $v(H) \geqslant \#(H) / p^{2}$ whenever $H \subseteq p^{2}$.

(e) $u_{3} \geqslant u_{6}$. This is Theorem $2 \mathrm{~F}$.

(f) $u_{6} \geqslant u_{1}$. Suppose that $\nu \in M_{1}\left(\mathscr{P} \omega^{2 \uparrow}\right)$ and $w(\nu)>u_{6}$.

By Lemma 2I there is a Radon probability $\mu$ on $\omega^{*}$ such that

$$
\nu\{\Gamma: \zeta(\Gamma, \mu) \geqslant w(\nu)\}>0 \text {. }
$$

Now

$$
\nu\left(S_{\Delta}\right) \geqslant \nu^{*}\left\{\Gamma: \zeta_{0}(\Gamma)>u_{6}\right\} \geqslant \nu\{\Gamma: \zeta(\Gamma, \mu) \geqslant w(\nu)\}>0 .
$$

As $\nu$ is arbitrary, $u_{1} \leqslant u_{6}$.

(g) $u_{1}=u_{7}$. Of course $u_{1}=u_{0} \geqslant u_{7}$. On the other hand, if $u<u_{0}=u_{4}$, there is a finite relation $H$, not admitting $\Delta$, with $v(H) \geqslant u$. In this case there is some $p<\omega$ such that $H$ does not admit $\Delta \cap p^{2}$, and $u_{7} \geqslant u_{0}\left(\Delta \cap p^{2}\right)=u_{4}\left(\Delta \cap p^{2}\right) \geqslant v(H) \geqslant$ $u$. As $u$ is arbitrary, $u_{7} \geqslant u_{0}$.

(h) $u_{1}=u_{2}$. Of course $u_{1} \geqslant u_{2}$ as $S_{\Delta}^{*} \supseteq S_{\Delta}$. On the other hand, if $u<u_{1}$, there is a finite relation $H$, not admitting $\Delta$, with $v(H) \geqslant u$. Let $\Delta^{\prime} \subseteq \Delta$ be a finite set such that $H$ does not admit $\Delta^{\prime}$. Then

$$
S_{\Delta^{\prime}}^{*}=\bigcup_{f \in F} T_{f^{\rightarrow}\left(\Delta^{\prime}\right)},
$$

where $F=\omega^{V\left(\Delta^{\prime}\right)}$ is countable. But since $H$ does not admit $f^{\rightarrow}\left(\Delta^{\prime}\right)$ for any $f \in F$ (since $g \rightarrow\left(f \rightarrow\left(\Delta^{\prime}\right)\right)=(g f)^{\rightarrow}\left(\Delta^{\prime}\right)$ cannot be a subset of $H$ for any $g: \omega \rightarrow V(H)$ ), $\lambda_{\chi H}\left(T_{f \rightarrow\left(\Delta^{\prime}\right)}\right)=0$ for every $f \in F$, where $\lambda_{\chi H} \in M_{1}\left(\mathscr{P} \omega^{2 \uparrow}\right)$ is defined as in (c) of this proof. So $\lambda_{\chi H}\left(S_{\Delta^{\prime}}^{*}\right)=0$. As $w\left(\lambda_{\chi H}\right)=v(H) \geqslant u, u_{2} \geqslant u$. As $u$ is arbitrary, $u_{2} \geqslant u_{1}$.

This completes the proof.

3. Finite subgraphs. Working with $u_{4}(\Delta)$, as described in Theorem $2 \mathrm{~J}$, we see that all our problems can be reduced to the study of finite relations $H$ and the graphs which they admit. In this section we show that, for finite $\Delta, u_{0}(\Delta)$ can be found with a finite amount of calculation, whose length we can bound (3D). We give a couple of special cases $(3 \mathrm{~F}, 3 \mathrm{G})$, and then describe the algorithm we have devised to calculate $u_{0}$ in practice $(3 \mathrm{H}-3 \mathrm{~L})$, with examples $(3 \mathrm{M})$. Our method is based on the concept of "critical matrix" (3A); we cannot believe that this is a new idea, but we have not been able to find a reference.

3A. Definition. Let $I$ be a nonempty finite set and let $\mathbf{A}=\left\langle a_{i j}\right\rangle_{i, j \in I} \in \mathbf{R}^{I \times I}$ be a real matrix.

(a) We set

$$
v(\mathbf{A})=\sup \left\{\sum_{i, j \in I} a_{i j} x_{i} x_{j}: x_{i} \geqslant 0 \forall i \in I, \sum_{i \in I} x_{i}=1\right\},
$$

and call $v(\mathbf{A})$ the value of $\mathbf{A}$. 
(b) We say that $\mathbf{A}$ is critical if $v(\mathbf{A})>v\left(\mathbf{A} \uparrow J^{2}\right)=v\left(\left\langle a_{i j}\right\rangle_{i, j \in J}\right)$ for every nonempty proper subset $J$ of $I$. (In particular, if $\#(I)=1$, then every $\mathbf{A} \in \mathbf{R}^{I \times I}$ is critical.)

(c) We say that $\mathbf{A}$ is pseudo-critical if there are unique $\lambda \in \mathbf{R},\left\langle x_{i}\right\rangle_{i \in I} \in \mathbf{R}^{I}$ such that

$$
\sum_{i \in I} x_{i}=1, \quad \sum_{j \in I}\left(a_{i j}+a_{j i}\right) x_{j}=\lambda \quad \forall i \in I,
$$

and, moreover, $\mathbf{x}=\left\langle x_{i}\right\rangle_{i \in I}$ is such that $x_{i}>0$ for every $i \in I$. In this case we say that $\mathbf{x}$ is the vector of natural weights for $\mathbf{A}$, and that $\lambda$ is the multiplier for $\mathbf{A}$.

(d) If $H$ is a nonempty finite relation, we say that the matrix associated with $H$ is the matrix $\mathbf{A}=\left\langle a_{i j}\right\rangle_{i, j \in V(H)}$ such that $a_{i j}=1$ if $(i, j) \in H$ and $a_{i j}=0$ if $(i, j) \in$ $V(H)^{2} \backslash H$. Observe that in this case $v(H)$, as defined in $1 \mathrm{~A}(\mathrm{i})$, is equal to $v(\mathbf{A})$ as defined in (a) above. We will say that $H$ is critical or pseudo-critical if $\mathbf{A}$ is. It seems appropriate to regard the empty relation as both critical and pseudo-critical.

3B. Proposition. Let $\mathbf{A} \in \mathbf{R}^{I \times I}$ be a matrix, where I is a nonempty set.

(a) $A$ is critical iff it is pseudo-critical and $\sum_{i, j \in I} a_{i j} z_{i} z_{j}<0$ whenever $\mathbf{z} \in \mathbf{R}^{I} \backslash\{\mathbf{0}\}$ and $\sum_{i \in I} z_{i}=0$.

(b) In this case, (i) if $x$ is the vector of natural weights for $\mathbf{A}$ and $\lambda$ is its multiplier,

$$
v(\mathbf{A})=\sum_{i, j \in I} a_{i j} x_{i} x_{j}=\frac{1}{2} \lambda ;
$$

(ii) if $k$, l are distinct members of $I$, then $a_{k k}+a_{l l}<a_{k l}+a_{l k}$.

Proof. For $\mathbf{y} \in \mathbf{R}^{I}$ set

$$
\varphi(\mathbf{y})=\mathbf{y}^{T} \mathbf{A y}=\sum_{i, j \in I} a_{i j} y_{i} y_{j} .
$$

Write $C=\left\{\mathbf{y}: \mathbf{y} \in \mathbf{R}^{I}, \sum_{i \in I} y_{i}=1\right\}, D=\{\mathbf{y}: \mathbf{y} \in C, \mathbf{y} \geqslant \mathbf{0}\}, \partial D=\{\mathbf{y}: \mathbf{y} \in D$, $\left.\exists i \in I, y_{i}=0\right\}$; define $\mathbf{e} \in \mathbf{R}^{I}$ by setting $e_{i}=1$ for every $i \in I$.

Suppose first that $\mathbf{A}$ is critical. As $\varphi$ is continuous and $D$ is compact, there is an $\mathbf{x} \in D$ such that $\varphi(\mathbf{x})=\max _{\mathbf{y} \in D} \varphi(\mathbf{y})=v(\mathbf{A})$. As $\mathbf{A}$ is critical, $\varphi(\mathbf{x})>\varphi(\mathbf{y})$ for every $\mathbf{y} \in \partial D$; because if $y_{i}=0$,

$$
\varphi(\mathbf{y}) \leqslant v\left(A \uparrow(I \backslash\{i\})^{2}\right)<v(\mathbf{A}) .
$$

So $\mathbf{x} \in D \backslash \partial D$.

Now take any $\mathbf{z} \in \mathbf{R}^{I}$ such that $\mathbf{e}^{T} \mathbf{z}=0$ and $\mathbf{z} \neq \mathbf{0}$, and for $\delta \in \mathbf{R}$ consider

$$
f_{\mathbf{z}}(\delta)=\varphi(\mathbf{x}+\delta \mathbf{z})=\varphi(\mathbf{x})+\delta \mathbf{x}^{T}\left(\mathbf{A}+\mathbf{A}^{T}\right) \mathbf{z}+\delta^{2} \mathbf{z}^{T} \mathbf{A z} \text {. }
$$

Then $f_{\mathbf{z}}$ is a quadratic and $f_{\mathbf{z}}(\delta) \leqslant f_{\mathbf{z}}(0)$ for every $\delta$ close enough to 0 , since $\mathbf{x}+\delta \mathbf{z} \in D$ for all $\delta$ close enough to 0 ; it follows that $\mathbf{x}^{T}\left(\mathbf{A}+\mathbf{A}^{T}\right) \mathbf{z}=f^{\prime}(0)=0$. Moreover, since there is some $\delta$ such that $\mathbf{x}+\delta \mathbf{z} \in \partial D, f_{\mathbf{z}}$ cannot be constant, and $\mathbf{z}^{T} \mathbf{A z}<0$.

Thus $\mathbf{x}^{T}\left(\mathbf{A}+\mathbf{A}^{T}\right) \mathbf{z}=0$ whenever $\mathbf{e}^{T} \mathbf{z}=0$. It follows at once that $\left(\mathbf{A}+\mathbf{A}^{T}\right) \mathbf{x}=\lambda \mathbf{e}$ for some $\lambda \in \mathbf{R}$; that is, that

$$
\sum_{j \in I}\left(a_{i j}+a_{j i}\right) x_{j}=\lambda \quad \forall i \in I .
$$


So we see that $\mathbf{x}$ and $\lambda$ satisfy the defining conditions of $3 \mathrm{~A}(\mathrm{c})$. To see that they are the only solutions, let $\mathbf{w}$ and $\lambda^{\prime}$ be an alternative pair satisfying the conditions; then we see that

$$
\varphi(\mathbf{w}+\mathbf{z})=\varphi(\mathbf{w})+\mathbf{z}^{T} \mathbf{A z}<\varphi(\mathbf{w})
$$

whenever $\mathbf{e}^{T} \mathbf{z}=0$ and $\mathbf{z} \neq \mathbf{0}$; in particular, considering $\mathbf{z}=\mathbf{x}-\mathbf{w}$, we must have $\mathbf{x}=\mathbf{w}$ and $\lambda=\lambda^{\prime}$.

Accordingly $\mathbf{A}$ is pseudo-critical with natural weights $\mathbf{x}$ and multiplier $\lambda$. We can compute $\lambda$ by saying

$$
\lambda=\sum_{i \in I} x_{i} \lambda=\sum_{i, j \in I}\left(a_{i j}+a_{j i}\right) x_{i} x_{j}=2 v(\mathbf{A}) .
$$

If $k$ and $l$ are distinct members of $I$, then consider $\mathbf{z} \in \mathbf{R}^{I}$ defined by writing

$$
z_{k}=1, \quad z_{l}=-1, \quad z_{i}=0 \quad \forall i \in I \backslash\{k, l\} .
$$

Then

$$
0>\mathbf{z}^{T} \mathbf{A z}=a_{k k}+a_{l l}-a_{k l}-a_{l k} .
$$

The arguments above prove one of the implications in (a) and the whole of (b). For the other half of (a), suppose that $\mathbf{A}$ is pseudo-critical and that $\mathbf{z}^{T} \mathbf{A z}<0$ whenever $\mathbf{e}^{T} \mathbf{z}=0$ and $\mathbf{z} \neq \mathbf{0}$. Let $\mathbf{x}$ be the vector of natural weights for $\mathbf{A}$ and $\lambda$ its multiplier, so that $\left(\mathbf{A}+\mathbf{A}^{T}\right) \mathbf{x}=\lambda \mathbf{e}$. Then the same calculations as those above show that $\varphi(\mathbf{x}+\mathbf{z})<\varphi(\mathbf{x})$ whenever $\mathbf{z}$ is nonzero and $\mathbf{e}^{T} \mathbf{z}=0$. Of course $v(\mathbf{A}) \geqslant \varphi(\mathbf{x})$. Now if $J$ is any nonempty proper subset of $I$, there is a $\mathbf{y} \in D$ such that $v\left(A \uparrow J^{2}\right)=\varphi(\mathbf{y})$ and $y_{i}=0$ for $i \in I \backslash J$; in this case, as $x_{i}>0$ for every $i \in I$, $\mathbf{z}=\mathbf{y}-\mathbf{x} \neq \mathbf{0}$, and

$$
v\left(A \uparrow J^{2}\right)=\varphi(\mathbf{x}+\mathbf{z})<\varphi(\mathbf{x}) \leqslant v(\mathbf{A}) .
$$

As $J$ is arbitrary, $\mathbf{A}$ is critical. This completes the proof.

3C. Lemma. Suppose that $1 \leqslant p<\omega$ and that $I$ is a finite set with $\#(I) \geqslant 2^{p-1}$. Let $H \subseteq I^{2}$ be such that for every distinct $k, l \in I$, at least one of $(k, l),(l, k)$ belongs to $H$. Then $H$ admits $p^{2 \uparrow}$.

Proof. We induce on $p$. For $p=1$ this is trivial. For the inductive step to $p+1$, let $I$ be a set of cardinal $2^{p}$, and $H \subseteq I^{2}$ a set satisfying the condition. We can suppose that $(i, i) \notin H$ for every $i \in I$. As

$$
\#(H) \geqslant 2^{p-1}\left(2^{p}-1\right)>2^{p}\left(2^{p-1}-1\right),
$$

there is a $k \in I$ such that $\#(J) \geqslant 2^{p-1}$, where $J=\{i:(i, k) \in H\}$. Now $H \cap J^{2}$ satisfies the condition of the lemma, so, by the inductive hypothesis, admits $p^{2 \uparrow}$; let $f: p \rightarrow J$ be such that $(f(i), f(j)) \in H$ whenever $i<j<p$. In this case we can extend $f$ to $g: p+1 \rightarrow I$ by saying that $g(p)=k$, and we see that $g \rightarrow\left((p+1)^{2 \uparrow}\right)$ $\subseteq H$. Thus the induction continues.

3D. THEOREM. Let $\Delta \subseteq p^{2 \uparrow}$, where $2 \leqslant p<\omega$.

(a) If $\Delta$ is not empty, there is a relation $H$ such that $H$ does not admit $\Delta$, $v(H)=u_{0}(\Delta)$, and $\#(V(H))<2^{p-1}$. Consequently $\left(\omega, 2, u_{0}(\Delta)\right) \nRightarrow \Delta$.

(b) If $\Delta$ has more than one member, there is a $\nu \in M_{1}\left(\mathscr{P} \omega^{2 \uparrow}\right)$ such that $\nu\left(U_{i j}\right)>$ $u_{0}(\Delta)$ whenever $i<j$ and $\nu\left(S_{\Delta}\right)=0$. 
Proof. (a) Set $u_{0}=u_{0}(\Delta)$, and let $u_{4}^{\prime}$ be $\max \{v(H)$ : $H$ is a relation not admitting $\Delta$, \#(V(H))<2 $\left.2^{p^{-1}}\right\}$. (The maximum is of course obtained.) Suppose, if possible, that $u_{4}^{\prime} \neq u_{0}(\Delta)$. By Theorem $2 \mathrm{~J}$ this means that there is a finite relation $H$, not admitting $\Delta$, such that $v(H)>u_{4}^{\prime}$. Set $I=V(H)$, and let $\mathbf{A}=\left\langle a_{i j}\right\rangle_{i, j \in I}$ be the matrix associated with $H(3 \mathrm{~A}(\mathrm{~d}))$. Let $J$ be a minimal nonempty subset of $I$ for which $v\left(\mathbf{A} \uparrow J^{2}\right)=v(\mathbf{A})$; then $\mathbf{A} \uparrow J^{2}$ is critical, and $v\left(\mathbf{A} \uparrow J^{2}\right)>u_{4}^{\prime}$.

Consider $H^{\prime}=H \cap J^{2}$. Then $H^{\prime}$ does not admit $\Delta$, and $v\left(H^{\prime}\right)=v\left(\mathbf{A} \uparrow J^{2}\right)>u_{4}^{\prime}$, so $\#(J) \geqslant 2^{p-1}$. On the other hand, if $k, l$ are distinct members of $J, a_{k k}+a_{k l}<$ $a_{k l}+a_{l k}$, by $3 \mathrm{~B}(\mathrm{c})$, because $\mathrm{A} \uparrow J^{2}$ is critical; it follows at once that at least one of $(k, l),(l, k)$ belongs to $H^{\prime}$. So $H^{\prime}$ admits $p^{2 \uparrow}$, by $3 \mathrm{C}$, which is impossible, because $\Delta \subseteq p^{2 \uparrow}$.

Thus $u_{0}=u_{4}^{\prime}$, and there is indeed a finite relation $H$, not admitting $\Delta$, with $v(H)=u_{0}$. Now if $H \neq \varnothing$, take $\nu=\lambda_{\chi H}$, as in part (c) of the proof of $2 \mathrm{~J}$; if $H=\varnothing$ take $\nu(\{\varnothing\})=1$. In either case $w(\nu)=u_{0}$ and $\nu\left(S_{\Delta}\right)=0$, so that $\left(\omega, 2, u_{0}\right)$ $\Rightarrow \Delta$.

(b) If $\Delta$ has two or more members we can refine this result, as follows. Let $\left\langle\alpha_{i j}\right\rangle_{i<j<\omega}$ be a family of strictly positive real numbers such that $\sum_{i<j<\omega} \alpha_{i j}=1$.

(i) If $u_{0}=0$ define $\nu \in M_{1}\left(\mathscr{P} \omega^{2 \uparrow}\right)$ by saying that

$$
\nu(\{(i, j)\})=\alpha_{i j} \quad \forall i<j<\omega .
$$

Then $\nu\left(U_{i j}\right)=\alpha_{i j}>0$ for each $i<j<\omega$, while $\nu\left(S_{\Delta}\right)=0$ because $\#(\Delta) \geqslant 2$.

(ii) If $u_{0}>0$, take $H$ from part (a). Let $I=V(H)$ and let $\mathbf{A} \in\{0,1\}^{I \times I}$ be the matrix corresponding to $H$. Let $\mathbf{z}$ be the vector of natural weights for $\mathbf{A}$. Then each $z_{i}$ is rational, because all the coefficients of $\mathbf{A}$ are rational; say $z_{i}=k_{i} / m$ for $i \in I$, where $0<k_{i}<m<\omega$. Imagine that each $i \in V(H)$ is divided into $k_{i}$ pieces; this gives us an $H^{\prime} \subseteq m^{2}$ such that

$$
\#\left(H^{\prime}\right)=\sum_{(i, j) \in H} k_{i} k_{j}=m^{2} v(H)=m^{2} u_{0},
$$

while $H^{\prime}$ does not admit $\Delta$ (because $H^{\prime} \preccurlyeq H$ ). For $k<l<\omega$ we construct $\nu_{k l} \in$ $M_{1}\left(\mathscr{P} \omega^{2 \uparrow}\right)$ by a method similar to that of $1 \mathrm{G}$. Set $X=m^{\omega}$ and identify $X$ with $m^{\omega \backslash\{k, l\}} \times m^{\{k, l\}}$. Give $m^{\omega \backslash\{k, l\}}$ the product measure obtained by giving each factor the uniform probability measure. Give $m^{\{k, l\}}$ the measure which gives mass $1 / m(m-1)$ to each point $(x, y)$ where $x, y<m$ and $x \neq y$. Give $X$ the product measure $\mu_{k l}$ and define $\varphi: X \rightarrow \mathscr{P} \omega^{2 \uparrow}$ by writing $\varphi(\mathbf{x})=\left\{(i, j): i<j<\omega,\left(x_{i}, x_{j}\right)\right.$ $\left.\in H^{\prime}\right\}$. Let $\nu_{k l}$ be the corresponding image measure in $M_{1}\left(\mathscr{P} \omega^{2 \uparrow}\right)$, as in $1 \mathrm{G}$. If $(i, j) \in \omega^{2 \uparrow}$ and $(i, j) \neq(k, l)$, then $\left(x_{i}, x_{j}\right)$ is uniformly distributed in $m^{2}$, so $\nu_{k l}\left(U_{i j}\right)=\#\left(H^{\prime}\right) / m^{2}=u_{0}$. On the other hand (because $H^{\prime}$ does not meet the diagonal of $\left.m^{2}\right), \nu_{k l}\left(U_{k l}\right)=\#\left(H^{\prime}\right) / m(m-1)=m u_{0} /(m-1)$, while $\nu_{k l}\left(S_{\Delta}\right)=0$ because $H^{\prime}$ does not admit $\Delta$.

Now take $\nu=\sum_{k<l<\omega} \alpha_{k l} \nu_{k l}$. Then $\nu\left(U_{i j}\right)=u_{0}\left(1+\alpha_{i j} /(m-1)\right)>u_{0}$ for every $i<j$, while $\nu\left(S_{\Delta}\right)=0$.

REMARKS. In the notation of [1], part (b) could be written " $\left(\omega, 2,>u_{0}\right) \neq \Delta$ ". Erdös and Hajnal prove this theorem for $\Delta=n^{2 \uparrow}$, and part (a) for $\Delta=\{(0,1)$, $(1,2)\}$; they leave part (b) for the latter as an open question. They speculate on the 
maximum possible value for $\sum_{i<j<\omega}\left(\nu\left(U_{i j}\right)-u_{0}\right)$ subject to $\nu\left(S_{\Delta}\right)=0$ and $\nu\left(U_{i j}\right)>$ $u_{0}$ for every $i, j$; our value is larger than theirs for $\Delta=n^{2 \uparrow}$; we do not know if it is best possible in this case.

We come now to two important special cases, one of which was already known. The first is simplified by the following characterization of critical relations of value less than $\frac{1}{2}$.

3E. Proposition. Let $H$ be a nonempty finite relation. Then the following are equivalent:

(i) $H$ is critical and $v(H)<\frac{1}{2}$;

(ii) $H$ is critical and there are no $i, j$ such that $(i, j)$ and $(j, i)$ both belong to $H$;

(iii) there is no $i$ such that $(i, i) \in H$ and whenever $i, j$ are distinct members of $V(H)$, exactly one of $(i, j),(j, i)$ belongs to $H$.

In this case $v(H)=(n-1) / 2 n$, where $n=\#(V(H))$.

Proof. (a) Easy calculations show that $v(\{(i, i)\})=1$ and $v(\{(i, j),(j, i)\})=\frac{1}{2}$ whenever $j \neq i$. Thus (i) $\Rightarrow$ (ii). From $3 \mathrm{~B}(\mathrm{c}$ ) we see that (ii) $\Rightarrow$ (iii).

(b) Suppose that $H$ satisfies (iii). Then it is easy to see that it is pseudo-critical, with all its natural weights equal to $1 / n$, and its multiplier equal to $(n-1) / n$. This shows that if $H$ satisfies either (i) or (ii), $v(H)=(n-1) / 2 n$; while if $H$ satisfies (iii), then $v(H) \geqslant(n-1) / 2 n$.

(c) Finally, again suppose that $H$ satisfies (iii). Then there is surely a $J \subseteq V(H)$ such that $H \cap J^{2}$ is critical and $v(H)=v\left(H \cap J^{2}\right)$. In this case, $H \cap J^{2}$ satisfies (ii) so $v(H)=(m-1) / 2 m$ where $m \leqslant \#(J)$. But as we already know that $v(H) \geqslant$ $(n-1) / 2 n, m=n$, so $H$ is indeed critical. Thus (iii) $\Rightarrow$ (i) and the proof is complete.

3F. Finite chains. We are now in a position to calculate $u_{0}(\Delta)$ where $\Delta$ is a simple finite chain. Write $\Delta_{C}=\{(i, i+1): i<\omega\}$, so that $\Delta_{C} \cap n^{2}$ is an ascending chain of $n-1$ links for $n \geqslant 2$.

(a) The essential idea is this: Suppose that $H \subseteq I^{2}$, where $\#(I) \geqslant n$, and for all distinct $i, j \in I$ at least one of $(i, j),(j, i)$ belongs to $H$; then $H$ admits $\Delta_{C} \cap n^{2}$. Our proof is by induction on $n$. For $n=1$ the result is trivial. For the inductive step to $n+1$, suppose that $\#(I)=n+1$, and take any $k \in I$. Set $J=I \backslash\{k\}$; then $H \cap J^{2}$ satisfies the condition in $J^{2}$, so, by the inductive hypothesis, $\Delta_{C} \cap n^{2} \leqslant H$ $\cap J^{2}$; let $f: n \rightarrow J$ be such that $(f(i), f(i+1)) \in H$ whenever $0 \leqslant i<n-1$. (i) If $f$ is not injective then $\Delta_{C} \preccurlyeq H$; for if $f(i)=f(j)$, where $i<j$, we can set

$$
g(k+r(j-i))=f(i+k)
$$

whenever $r<\omega, k<j-i$ and see that $g \rightarrow\left(\Delta_{C}\right) \subseteq H$. (ii) If $(f(n-1), k) \in H$ we can set $g(i)=f(i)$ for $i<n$ and $g(n)=k$, and see that $g \rightarrow\left(\Delta_{C} \cap(n+1)^{2}\right) \subseteq H$. (iii) If $(k, f(0)) \in H$ we can set $g(0)=k$ and $g(i+1)=f(i)$ for $i<n$, and see that $g \rightarrow\left(\Delta_{C} \cap(n+1)^{2}\right) \subseteq H$. (iv) If $f$ is injective and neither $(k, f(0))$ nor $(f(n-1), k)$ $\in H$, consider $K=\{i: i \leqslant n-2,(f(i), k) \in H\}, L=\{i: i \leqslant n-2,(k, f(i+1))$ $\in H\}$. Then

$$
\#(K)+\#(L)=\{j: j \neq k,(j, k) \in H\}+\#\{j: j \neq k,(k, j) \in H\} \geqslant n .
$$


So there is an $l \in K \cap L$; now setting $g(i)=f(i)$ for $i \leqslant l, g(l+1)=k$, and $g(i)=f(i-1)$ for $l+2 \leqslant i \leqslant n$, we once more have $g \rightarrow\left(\Delta_{C} \cap(n+1)^{2}\right) \subseteq H$. Thus in all cases the induction proceeds.

(b) It follows that $u_{0}\left(\Delta_{C} \cap n^{2}\right) \leqslant(n-2) / 2(n-1)$. For suppose that $H$ is a nonempty critical relation not admitting $\Delta_{C} \cap n^{2}$. Then surely we can never have both $(i, j)$ and $(j, i)$ belonging to $H$ for any $i, j$; for if this occurred then $f \rightarrow\left(\Delta_{C}\right)$ would be a subset of $H$, where $f(k)=i$ for even $k, j$ for odd $k$. So by $3 \mathrm{~K}$, $v(H)=(m-1) / 2 m$, where $m=\#(V(H))$. On the other hand, $m<n$ by part (a). So $v(H) \leqslant(n-2) / 2(n-1)$. By $2 \mathrm{~J}$ this gives the result.

(c) On the other hand, it is easy to see that $(n-1)^{2 \uparrow}$ does not admit $\Delta_{C} \cap n^{2}$, because there can be no strictly increasing function from $n$ to $n-1$. So $u_{0}\left(\Delta_{C} \cap n^{2}\right)$ $\geqslant v\left((n-1)^{2 \uparrow}\right)=(n-2) / 2(n-1)$ by $3 \mathrm{E}$.

(d) Thus $u_{0}\left(\Delta_{C} \cap n^{2}\right)=(n-2) / 2(n-1)$ for every $n \geqslant 2$.

REMARK. This result was conjectured in [1], where it was proved for $n=3$, and the inequality of (c) was proved for all $n$.

3G. Complete graphs. An argument in the same spirit as those above can be used to give the result, proved in [1], that $u_{0}\left(n^{2 \uparrow}\right)=(n-2) /(n-1)$ for $n \geqslant 2$. Here the basic idea is: Suppose that $H \subseteq I^{2}$ and $\#(H)>(n-2) \#(I)^{2} /(n-1)$; then $H$ admits $n^{2 \uparrow}$. As in $3 \mathrm{G}$, this is easily proved by induction on $n$. Consequently, using $u_{3}$ of Theorem $2 \mathrm{~J}, u_{0}\left(n^{2 \uparrow}\right) \leqslant(n-2) /(n-1)$. On the other hand, we find that

$$
H^{*}=\{(i, j): i, j<n-1, i \neq j\}
$$

does not admit $n^{2 \uparrow}$ (because there is no injective function from $n$ to $n-1$ ), and that $H^{*}$ is pseudo-critical, with natural weights $1 /(n-1)$ and multiplier

$$
2(n-2) /(n-1) \text {; }
$$

so that

$$
u_{0}\left(n^{2 \uparrow}\right) \geqslant v\left(H^{*}\right) \geqslant(n-2) /(n-1) .
$$

This argument is of course exactly parallel to P. Turán's theorem that if $D \subseteq[m]^{2}$ and $\#(D)>(n-2) m^{2} / 2(n-1)$, then $D \supseteq[I]^{2}$ for some $I \in[m]^{n}$, which is the basis of the argument in [1].

We now come to our methods for shorteningcalculation of $u_{0}(\Delta)$ for an arbitrary finite $\Delta$. They are based on the following concept.

3H. Definition. Let $\mathbf{B} \in R^{J \times J}$ be a pseudo-critical matrix, with multiplier $\lambda$ and natural weights $\mathbf{y}$. A simple improvement of $\mathbf{B}$ is a matrix $\mathbf{A} \in R^{I \times I}$, where $I \supseteq J$ and $I \backslash J$ is a singleton $\{k\}$ say, such that $\mathbf{B}=A \uparrow J^{2}$ and

$$
\sum_{i \in J}\left(a_{k i}+a_{i k}\right) y_{i}>\lambda \text {. }
$$

Similarly, if $K$ is a pseudo-critical relation, a simple improvement of $K$ is a relation $H$ such that the matrix associated with $H$ is a simple improvement of the matrix associated with $K$. (If $K=\varnothing$, an appropriate convention is to regard a relation $H$ as a simple improvement of $K$ iff $\#(V(H))=2$ and the matrix associated with $H$ is a simple improvement of a $1 \times 1$ zero matrix.) 
3I. Proposition. Let $\mathbf{B} \in \mathbf{R}^{J \times J}$ be a critical matrix and $\mathbf{A} \in \mathbf{R}^{I \times I}$ a simple improvement of $\mathbf{B}$. Then $v(\mathbf{A})>v(\mathbf{B})$. If $\mathbf{A}$ is pseudo-critical, it is critical.

Proof. Let $\left\langle y_{i}\right\rangle_{i \in J}$ be the vector of natural weights for $\mathbf{B}$; extend it to $\mathbf{y} \in \mathbf{R}^{I}$ by setting $y_{k}=0$, where $k$ is the member of $I \backslash J$. Define d, e $\in \mathbf{R}^{I}$ by setting

$$
\begin{array}{ll}
d_{k}=1, & d_{i}=0 \quad \forall i \in J, \\
e_{k}=0, & e_{i}=1 \quad \forall i \in J .
\end{array}
$$

Then 3B translates into

$$
\begin{aligned}
& \left(\mathbf{A}+\mathbf{A}^{T}\right) \mathbf{y}=2 v(\mathbf{B}) \mathbf{e}+\left(\mathbf{d}^{T}\left(\mathbf{A}+\mathbf{A}^{T}\right) \mathbf{y}\right) \mathbf{d}, \\
& \mathbf{z}^{T} \mathbf{A z}<0 \quad \text { whenever } \mathbf{z} \in \mathbf{R}^{I} \backslash\{\mathbf{0}\} \text { and } \mathbf{d}^{T} \mathbf{z}=\mathbf{e}^{T} \mathbf{z}=0, \\
& v(\mathbf{B})=\mathbf{y}^{T} \mathbf{A y} ;
\end{aligned}
$$

and $3 \mathrm{H}$ translates into

$$
\mathbf{d}^{T}\left(\mathbf{A}+\mathbf{A}^{T}\right) \mathbf{y}>2 v(\mathbf{B}) .
$$

(a) To estimate $v(\mathbf{A})$ set

$$
f(\delta)=((1-\delta) \mathbf{y}+\delta \mathbf{d})^{T} \mathbf{A}((1-\delta) \mathbf{y}+\delta \mathbf{d}) .
$$

Easy calculations show that

$$
f(0)=v(\mathbf{B}), \quad f^{\prime}(0)=\mathbf{d}^{T}\left(\mathbf{A}+\mathbf{A}^{T}\right) \mathbf{y}-2 \mathbf{y}^{T} \mathbf{A y}>0 .
$$

So

$$
v(\mathbf{A}) \geqslant \sup _{0 \leqslant \delta \leqslant 1} f(\delta)>v(\mathbf{B}) .
$$

(b) Now suppose that $\mathbf{A}$ is pseudo-critical; let $\mathbf{x}$ be its vector of natural weights and $\lambda$ its multiplier, so that

$$
\left(\mathbf{A}+\mathbf{A}^{T}\right) \mathbf{x}=\lambda(\mathbf{d}+\mathbf{e}) .
$$

We first have to note that $\mathbf{x}^{T} \mathbf{A x}>v(\mathbf{B})$. To see this, observe that $\mathbf{x}$ is expressible as $\left(1-x_{k}\right)(\mathbf{y}+\mathbf{u})+x_{k} \mathbf{d}$, where $\mathbf{d}^{T} \mathbf{u}=\mathbf{e}^{T} \mathbf{u}=0$ and $x_{k}>0$. So

$$
\begin{aligned}
2 \mathbf{x}^{T} \mathbf{A} \mathbf{x} & =\lambda \mathbf{x}^{T}(\mathbf{d}+\mathbf{e})=\lambda=\lambda \mathbf{y}^{T}(\mathbf{d}+\mathbf{e})=\mathbf{y}^{T}\left(\mathbf{A}+\mathbf{A}^{T}\right) \mathbf{x} \\
& =\left(\left(1-x_{k}\right)(\mathbf{y}+\mathbf{u})^{T}+x_{k} \mathbf{d}^{T}\right)\left(\mathbf{A}+\mathbf{A}^{T}\right) \mathbf{y} \\
& =2 v(\mathbf{B})\left(1-x_{k}\right)(\mathbf{y}+\mathbf{u})^{T} \mathbf{e}+x_{k} \mathbf{d}^{T}\left(\mathbf{A}+\mathbf{A}^{T}\right) \mathbf{y} \\
& =2 v(\mathbf{B})+x_{k}\left(\mathbf{d}^{T}\left(\mathbf{A}+\mathbf{A}^{T}\right) \mathbf{y}-2 v(\mathbf{B})\right)>2 v(\mathbf{B}),
\end{aligned}
$$

which is what we need.

Let $\mathbf{z} \in \mathbf{R}^{I} \backslash\{\boldsymbol{0}\}$ be such that $(\mathbf{d}+\mathbf{e})^{T} \mathbf{z}=0$. If $\mathbf{d}^{T} \mathbf{z}=0$ then certainly $\mathbf{z}^{T} \mathbf{A z}<0$. Otherwise, set $\alpha=z_{k} / x_{k}$ and $\mathbf{w}=\mathbf{x}-\alpha^{-1} \mathbf{z}$, so that $\mathbf{z}=\alpha(\mathbf{x}-\mathbf{w})$ and $\mathbf{d}^{T} \mathbf{w}=0$, 
$\mathbf{e}^{T} \mathbf{w}=1$. In this case

$$
\begin{aligned}
\mathbf{z}^{T} \mathbf{A z} & =\alpha^{2}\left(\mathbf{x}^{T} \mathbf{A} \mathbf{x}-\mathbf{w}^{T}\left(\mathbf{A}+\mathbf{A}^{T}\right) \mathbf{x}+\mathbf{w}^{T} \mathbf{A} \mathbf{w}\right) \\
& \leqslant \alpha^{2}\left(\mathbf{x}^{T} \mathbf{A} \mathbf{x}-\lambda \mathbf{w}^{T}(\mathbf{d}+\mathbf{e})+v(\mathbf{B})\right) \\
& =\alpha^{2}\left(-\mathbf{x}^{T} \mathbf{A} \mathbf{x}+v(\mathbf{B})\right)<0 .
\end{aligned}
$$

As $\mathbf{z}$ is arbitrary, $\mathbf{A}$ is critical, by $3 \mathrm{~B}(\mathrm{a})$.

3J. Proposition. Let I be a finite set with $\#(I)=n \geqslant 2$, and $\mathbf{A} \in \mathbf{R}^{I \times I}$ a critical matrix. Then there is a set $J \subseteq I$ such that $\#(J)=n-1, \mathbf{B}=\mathbf{A} \uparrow J^{2}$ is critical, $v(\mathbf{B}) \geqslant v\left(\mathbf{A} \uparrow K^{2}\right)$ for every nonempty proper subset $K$ of $I$, and $\mathbf{A}$ is a simple improvement of $\mathbf{B}$. If $a_{i i} \geqslant 0$ for every $i \in I$, then

$$
v(\mathbf{B}) \geqslant \frac{n(n-2)}{(n-1)^{2}} v(\mathbf{A}) .
$$

Proof. Let $\mathbf{x}$ be the vector of natural weights for $\mathbf{A}$, and set $e_{i}=1$ for $i \in I$. Then $\left(\mathbf{A}+\mathbf{A}^{T}\right) \mathbf{x}=2 v(\mathbf{A}) \mathbf{e}$.

(a) Let $J$ be a proper nonempty subset of $I$ for which $v\left(\mathbf{A} \uparrow J^{2}\right)$ is maximal; if there is more than one such, take $J$ to be as small as possible. Then $\mathbf{B}=\mathbf{A} \uparrow J^{2}$ is critical. So we can speak of the vector $\left\langle y_{i}\right\rangle_{i \in J}$ of natural weights for $\mathbf{B}$; define $\mathbf{y} \in \mathbf{R}^{I}$ by taking $y_{i}=0$ for $i \in I \backslash J$. Define $\mathbf{w}, \mathbf{z} \in \mathbf{R}^{I}$ by requiring $\mathbf{x}=\mathbf{w}+\mathbf{z}$ and $z_{i}=0$ for $i \in J, w_{i}=0$ for $i \in I \backslash J$.

We need to compute

$$
\begin{aligned}
\mathbf{z}^{T}\left(\mathbf{A}+\mathbf{A}^{T}\right) \mathbf{y} & =\mathbf{x}^{T}\left(\mathbf{A}+\mathbf{A}^{T}\right) \mathbf{y}-\mathbf{w}^{T}\left(\mathbf{A}+\mathbf{A}^{T}\right) \mathbf{y} \\
& =2 v(\mathbf{A}) \mathbf{e}^{T} \mathbf{y}-2 v(\mathbf{B}) \mathbf{w}^{T} \mathbf{e} \\
& =2 v(\mathbf{A})-2 v(\mathbf{B})\left(1-\mathbf{z}^{T} \mathbf{e}\right)>2 v(\mathbf{B}) \mathbf{z}^{T} \mathbf{e}
\end{aligned}
$$

So there is a $k \in I$ such that

$$
z_{k} \sum_{j \in I}\left(a_{k j}+a_{j k}\right) y_{j}>2 v(\mathbf{B}) z_{k}
$$

In this case $k$ must belong to $I \backslash J$ and $\mathbf{C}=\mathbf{A} \uparrow K^{2}$ is a simple improvement of $\mathbf{B}$, where $K=J \cup\{k\}$. By $3 \mathrm{I}(\mathrm{a}), v(\mathbf{C})>v(\mathbf{B})$. So $K$ cannot be a proper subset of $I$; thus $\mathbf{C}=\mathbf{A}$ and $\#(J)=n-1$, and $\mathbf{A}$ is a simple improvement of $\mathbf{B}$.

(b) If now $a_{i i} \geqslant 0$ for every $i \in I$, we can obtain a lower bound for $v(\mathbf{B})$, as follows. Let $l \in I$ be such that $x_{l} \leqslant 1 / n$, and consider $L=I \backslash\{l\}, \mathbf{D}=\mathbf{A} \uparrow L^{2}$. We must have $v(\mathbf{B}) \geqslant v(\mathbf{D})$. On the other hand,

$$
v(\mathbf{D}) \geqslant \sum_{i, j \in L} a_{i j} \frac{x_{i}}{1-x_{l}} \frac{x_{j}}{1-x_{l}},
$$


so that

$$
\begin{aligned}
v(\mathbf{A}) & =\sum_{i, j \in L} a_{i j} x_{i} x_{j}+\sum_{i \in I}\left(a_{i l}+a_{l i}\right) x_{i} x_{l}-a_{l l} x_{l}^{2} \\
& \leqslant\left(1-x_{l}\right)^{2} v(\mathbf{D})+2 v(\mathbf{A}) x_{l}
\end{aligned}
$$

because $a_{\|} \geqslant 0$. Accordingly

$$
v(\mathbf{B}) \geqslant v(\mathbf{D}) \geqslant \frac{1-2 x_{l}}{\left(1-x_{l}\right)^{2}} \geqslant \frac{1-2 / n}{(1-1 / n)^{2}}=\frac{n(n-2)}{(n-1)^{2}},
$$

as claimed.

3K. Corollary. Let $H$ be a nonempty critical relation. Then there is an increasing sequence $\left\langle I_{r}\right\rangle_{1 \leqslant r \leqslant n}$ of subsets of $V(H)$, where $n=\#(V(H))$, such that

(i) $\#\left(I_{r}\right)=r$ for $1 \leqslant r \leqslant n$;

(ii) $H \cap I_{r}^{2}$ is critical for $1 \leqslant r \leqslant n$;

(iii) $H \cap I_{r+1}^{2}$ is a simple improvement of $H \cap I_{r}^{2}$ for $1 \leqslant r<n$;

(iv)

$$
v\left(H \cap I_{r}^{2}\right) \geqslant \frac{(r-1) n}{r(n-1)} v(H) \quad \text { for } 1 \leqslant r \leqslant n .
$$

Proof. This is an elementary induction on $n$, using $3 \mathrm{~J}$ for the inductive step.

3L. The Algorithm. We now describe the basis of our computer program to calculate $u_{0}(\Delta)$. Take a finite $\Delta \subseteq \omega^{2 \uparrow}$. At each stage in the calculation we have a finite list $H_{0}, H_{1}, \ldots, H_{m}$ of critical relations not admitting $\Delta$, and a lower bound $u^{*}=\max _{i \leqslant m} v\left(H_{i}\right)$ for $u_{0}$. The program extends the list by taking one of the $H_{i}$ and adding isomorphic copies of all its pseudo-critical simple improvements which do not admit $\Delta$ (by $3 \mathrm{I}(\mathrm{b})$ these are all in fact critical). Their values are calculated as half their multipliers $(3 \mathrm{~B}(\mathrm{~b})(\mathrm{i}))$. By $3 \mathrm{~B}(\mathrm{~b})(\mathrm{ii})$ and $3 \mathrm{C}$ we know that this process will terminate, since all sufficiently large critical relations admit $\Delta$. From $3 \mathrm{D}($ a) and $3 \mathrm{~K}$ we see that our $u^{*}$ will then be $u_{0}(\Delta)$, since the critical relation $H$ of $3 \mathrm{D}$ (a) must be obtainable at the end of a finite sequence of extensions of the type that the program seeks out. The decisions on which simple extensions are pseudo-critical are based on rational linear algebra which can be done exactly. We have devised a number of ways of shortening the calculation; one is provided by $3 \mathrm{~K}(\mathrm{iv})$, which shows that we can pass over any $H_{i}$ such that $v\left(H_{i}\right) \leqslant(r-1) u^{*} / r$, where $r=\#\left(V\left(H_{i}\right)\right)$; and 3B(b)(ii) also helps by showing that most of the simple improvements of $H_{i}$ cannot be critical, so need not be tested.

3M. Two examples. We illustrate the remarks above by describing two special cases, chosen for sentimental reasons, as those which led us to the ideas presented here.

(a) $\Delta=\{(0,1),(1,2),(2,3),(0,3)\}$.

Here $H^{*}=\{(i, j): i<j<3\}$ does not admit $\Delta$, so $u_{0}(\Delta) \geqslant v\left(H^{*}\right)=\frac{1}{3}$. But since we find that (i) the only critical simple improvements of $\varnothing$ are (up to isomorphism) $\{(0,1)\}$ and $\{(0,1),(1,0)\}$, and the latter admits $\Delta$, (ii) the only critical 
simple improvements of $\{(0,1)\}$ are (up to isomorphism) $H^{*}$ and $\{(0,1),(1,2)$, $(2,0)\}$, and (iii) all the simple improvements of these two admit $\Delta$ (this is the laborious part of the calculation), $u_{0}(\Delta)$ is indeed $\frac{1}{3}$ exactly.

(b) $\Delta=\{(0,1),(0,2),(1,2),(1,3),(2,3),(2,4)\}$.

Here $H^{*}=\{(0,1),(0,2),(1,0),(1,2),(2,0)\}$ does not admit $\Delta$, so $u_{0}(\Delta) \geqslant v(H)=$ $\frac{4}{7}$. In this case we find that (i) the only critical simple improvement of $\varnothing$ with value greater than $\frac{1}{2} \cdot \frac{4}{7}$ is (up to isomorphism) $\{(0,1),(1,0)\}$, (ii) the only critical simple improvements of this are (up to isomorphism) $H^{*}$ and $\{(i, j): i, j<3, i \neq j\}$, and the latter admits $\Delta$, and (iii) every simple improvement of $H^{*}$ admits $\Delta$, so $u_{0}(\Delta)$ is indeed $\frac{4}{7}$.

$3 \mathrm{~N}$. Remark. The calculation in $3 \mathrm{M}(\mathrm{a})$ gives us an example to distinguish $u_{0}(\Delta)$, as defined above, from an alternative concept. For any $\Gamma \subseteq \omega^{2 \uparrow}$ set

$$
\begin{aligned}
& d^{*}(\Gamma)=\limsup _{n \rightarrow \infty} 2 \#\left(\Gamma \cap n^{2}\right) / n^{2}, \\
& d_{*}(\Gamma)=\liminf _{n \rightarrow \infty} 2 \#\left(\Gamma \cap n^{2}\right) / n^{2},
\end{aligned}
$$

the upper and lower densities of $\Gamma$. We can now consider, at least for finite $\Delta$,

$$
\begin{aligned}
& u^{*}(\Delta)=\sup \left\{d^{*}(\Gamma): \Delta \nsubseteq \nsubseteq \Gamma\right\}, \\
& u_{*}(\Delta)=\sup \left\{d_{*}(\Gamma): \Delta \nsubseteq \nsubseteq \Gamma\right\} .
\end{aligned}
$$

If $\nu \in M_{1}\left(\mathscr{P} \omega^{2 \uparrow}\right)$ is $h$-representable, then $d^{*}(\Gamma)=d_{*}(\Gamma)=w(\nu)$ for $\nu$-almost all $\Gamma$; this is a version of the strong law of large numbers. So Theorem $2 \mathrm{~J}$ tells us that $u_{0}(\Delta) \leqslant u_{*}(\Delta) \leqslant u^{*}(\Delta)$. Turán's theorem shows that if $d^{*}(\Gamma)>(n-2) /(n-1)$ then $n^{2 \uparrow} \subseteq \Gamma$; so that $u^{*}\left(n^{2 \uparrow}\right)=u_{*}\left(n^{2 \uparrow}\right)=u_{0}\left(n^{2 \uparrow}\right)$ for $n \geqslant 2$. Now all we wish to say here is that it is possible to have $u_{0}(\Delta)<u_{*}(\Delta)$. To see this, take $\Delta=\{(0,1)$, $(1,2),(2,3),(0,3)\}$ as in $3 \mathrm{M}(\mathrm{a})$, and set

$$
\Gamma=\{(i, j): i, j<\omega, i \sqrt{3}<j \leqslant 3 i \sqrt{3}\} .
$$

Then it is easy to check that $\Delta \nsubseteq \subseteq \Gamma$ and $d_{*}(\Gamma)=2 / 3 \sqrt{3}$, so that $u_{*}(\Delta) \geqslant 2 / 3 \sqrt{3}>$ $1 / 3=u_{0}(\Delta)$.

4. Some infinite graphs. As we explained in $\S 3$, we have developed methods of calculating $u_{0}(\Delta)$, for finite $\Delta$, which are effective in that we have a computer program which produces answers for small graphs $\Delta$, and is limited in its applicability only by the size and speed of the machine running it. We do not have a general formula which can be applied to calculate $u_{0}(\Delta)=\lim _{n \rightarrow \infty} u_{0}\left(\Delta \cap n^{2}\right)$ for general infinite graphs $\Delta$. For the most interesting cases, however, we have found particular arguments, which we give below.

4A. Proposition. Let $\Delta$ be the graph $\{(2 i, 2 j+1): i<j<\omega\}$. Then $u_{0}(\Delta)=0$.

Proof. We see that $S_{\Delta}^{*}=\omega^{2 \uparrow} \backslash\{\varnothing\}$; now $u_{2}$ of $2 \mathrm{~J}$ gives the result.

4B. Corollary. Suppose that $\nu \in M_{1}\left(\mathscr{P} \omega^{2 \uparrow}\right)$ and $w(\nu)>0$. Then $\nu\{\Gamma: \exists$ injective $f: \omega \rightarrow \omega$ such that, for each $i<\omega$, either $(f(i+1), f(i)) \in \Gamma$ or $(f(i), f(i+1)) \in$ $\Gamma\}>0$.

Proof. For if $\Delta \subseteq \Gamma$, where $\Delta$ is the graph of $4 \mathrm{~A}$, there is such an injective function $f: \omega \rightarrow \omega$. 
4C. Theorem. Let $\Delta_{A}$ be $\{(i, j): i, j<\omega, i+j$ is odd $\}$.

(a) If $\Gamma \subseteq \omega^{2 \uparrow}$ and $\mu$ is a Radon probability on $\omega^{*}$ such that $\zeta(\Gamma, \mu) \geqslant \frac{1}{2}$, then $\Delta_{A} \subset \Gamma$.

(b) $\left(\omega, 2, \frac{1}{2}\right) \Rightarrow \Delta_{A}$.

Proof. (a) Construct $h: \omega^{* 2} \rightarrow[0,1]$ as in 2B. From Lemma $2 \mathrm{E}$ we see that we can suppose that there is some $E \subseteq \omega^{*}$ such that $\mu E>0$ and $\int_{E \times E} h=0$; since otherwise $\omega^{2 \uparrow} \subseteq \Gamma$. Also $\int h=\zeta(\Gamma, \mu) \geqslant \frac{1}{2}$. Now there are two possibilities. (i) Setting $F=\omega^{*} \backslash E$ and $H=\{(x, y): h(x, y)>0\}$, we may have

$$
\mu^{2}(H \cap(E \times F))+\mu^{2}(H \cap(F \times E))>\mu F \cdot \mu E \text {. }
$$

In this case

$$
\mu^{2}\{(x, y): x \in E, y \in F,(x, y) \in H,(y, x) \in H\}>0 .
$$

Since $\Delta_{A} \preccurlyeq\{(0,1),(1,0)\}$, Lemma $2 \mathrm{D}$ tells us that $\Delta_{A} \subseteq \Gamma$. (ii) Otherwise, since $\mu^{2} H \geqslant \frac{1}{2}$, we must have

$$
\mu^{2}(H \cap(F \times F))>\frac{1}{2}(\mu F)^{2} .
$$

As in (c) of the proof of $2 \mathrm{~F}$, we can suppose that $\mu^{2}(H \cap(G \times G))=0$ for every atom $G$ for $\mu$. But in this case (again using the arguments in $2 F$ ) we find that there are disjoint nonnegligible measurable sets $F_{0}, F_{1} \subseteq F$ such that

$$
\mu^{2}\left(H \cap\left(F_{0} \times F_{1}\right)\right)>\frac{1}{2} \mu F_{0} \mu F_{1}, \quad \mu^{2}\left(H \cap\left(F_{1} \times F_{0}\right)\right)>\frac{1}{2} \mu F_{1} \mu F_{0} ;
$$

and in this case

$$
\mu^{2}\left\{(x, y): x \in F_{0}, x \in F_{1},(x, y) \in H,(y, x) \in H\right\}>0,
$$

so that once again $\Delta_{A} \subseteq \Gamma$.

(b) Now if $\nu \in M_{1}\left(\mathscr{P} \omega^{2 \uparrow}\right)$ and $w(\nu) \geqslant \frac{1}{2}$, we see from 2 I that there is a Radon probability $\mu$ on $\omega^{*}$ such that

$$
\nu\{\Gamma: \zeta(\Gamma, \mu) \geqslant w(\nu)\}>0,
$$

so that $\nu\left\{\Gamma: \Delta_{A} \subseteq \Gamma\right\}>0$, as required.

4D. Corollary. $u_{0}\left(\Delta_{A}\right)=u_{0}\left(\Delta_{C}\right)=\frac{1}{2}$, where $\Delta_{A}$ is defined in $4 \mathrm{C}$ and $\Delta_{C}$ is defined in $3 \mathrm{~F}$.

Proof. We already know that $u_{0}\left(\Delta_{C}\right)=\sup _{n<\omega} u_{0}\left(\Delta_{C} \cap n^{2}\right)=\frac{1}{2}$, by $2 \mathrm{~J}$ and $3 \mathrm{~F}$. Now of course $u_{0}\left(\Delta_{C}\right) \leqslant u_{0}\left(\Delta_{A}\right)$ because $\Delta_{C} \subseteq \Delta_{A}$, and $u_{0}\left(\Delta_{A}\right) \leqslant \frac{1}{2}$ by $4 \mathrm{C}$.

5. $h$-representable measures. We show that the $h$-representable measures $(1 \mathrm{G})$ are precisely the extreme deletion-invariant measures. While we have no applications to offer of this result, it was an essential part of the process by which we arrived at Theorem $2 \mathrm{~J}$, and we feel that it remains illuminating.

5A. The measure defined by a graph and a sequence of sets. From here down to 5F we suppose that we have fixed

(i) a disjoint sequence $\left\langle I_{n}\right\rangle_{n<\omega}$ of nonempty finite subsets of $\omega$ such that $\max I_{n}<\min I_{n+1}$ for every $n<\omega$;

(ii) a nonprincipal ultrafilter $\mathscr{F}$ on $\omega$; 
(iii) the Radon measure $\mu$ on $\omega^{*}$ defined by writing

$$
\mu A^{*}=\lim _{n \rightarrow \mathscr{F}} \frac{\#\left(A \cap I_{n}\right)}{\#\left(I_{n}\right)} \quad \forall A \subseteq \omega
$$

(cf. 2I);

(iv) a graph $\Gamma \subseteq \omega^{2 \uparrow}$;

(v) a function $h: \omega^{* 2} \rightarrow[0,1]$ constructed from $\Gamma$ and $\mu$ as in 2B;

(vi) the measure $\lambda_{h} \in M_{1}\left(\mathscr{P} \omega^{2 \uparrow}\right)$ constructed from $h$ and $\mu$ as in $1 \mathrm{G}$;

(vii) a $p<\omega$ and a $\Delta \subseteq(p+1)^{2 \uparrow}$.

Our aim is to give a formula for $\lambda_{h}\left(T_{\Delta}\right)$ in terms of $\left\langle I_{n}\right\rangle_{n<\omega}, \Gamma, \mathscr{F}$ and $\Delta$. Our proof is complex and technical, though the underlying ideas are no deeper than those of §2. Our formulas will be simplified by writing

$$
q(B, a)=\mu(B \cap \Gamma[\{a\}])^{*} \quad \forall a<\omega, B \subseteq \omega .
$$

Then we see that the defining formula for $h$ becomes

$$
\int_{A^{*} \times B^{*}} h=\int g_{A B}^{*} \quad \forall A, B \subseteq \omega,
$$

where $g_{A B}(a)=q(B, a)$ if $a \in A, 0$ if $a \in \omega \backslash A$; so that

$$
\int_{A^{*} \times B^{*}} h=\lim _{n \rightarrow \mathscr{F}} \frac{1}{\#\left(I_{n}\right)} \sum_{a \in I_{n} \cap A} q(B, a) \quad \forall A, B \subseteq \omega
$$

(cf. 2I).

We start with an elementary remark which we shall use repeatedly.

5B. Lemma. If $\boldsymbol{\delta}_{0}, \ldots, \boldsymbol{\delta}_{k}, \delta_{0}^{\prime}, \ldots, \boldsymbol{\delta}_{k}^{\prime} \in \mathbf{R}$ then

$$
\left|\prod_{i \leqslant k} \delta_{i}-\prod_{i \leqslant k} \delta_{i}^{\prime}\right| \leqslant \sum_{i \leqslant k}\left(\left|\delta_{i}-\delta_{i}^{\prime}\right| \cdot \prod_{\substack{j \leqslant k \\ j \neq i}} \max \left(\left|\delta_{j}\right|,\left|\delta_{j}^{\prime}\right|\right)\right) .
$$

Proof. Induce on $k$.

5C. Lemma. If $A, B \subseteq \omega$ and $\alpha \in \mathbf{R}$ then

$$
\lim _{n \rightarrow \mathscr{F}} \frac{1}{\#\left(I_{n}\right)} \sum_{a \in I_{n} \cap A}\left|\alpha \mu B^{*}-q(B, a)\right| \leqslant \int_{A^{*} \times B^{*}}|h(x, y)-\alpha| d x d y .
$$

Proof. Set $C=\left\{a: a \in A, \alpha \mu B^{*}>q(B, a)\right\}, D=A \backslash C$. Then the left-hand expression is

$$
\begin{aligned}
\lim _{n \rightarrow \mathscr{F}} & \frac{1}{\#\left(I_{n}\right)} \sum_{a \in I_{n} \cap C}\left(\alpha \mu B^{*}-q(B, a)\right)+\lim _{n \rightarrow \mathscr{F}} \frac{1}{\#\left(I_{n}\right)} \sum_{a \in I_{n} \cap D}\left(q(B, a)-\alpha \mu B^{*}\right) \\
& =\alpha \mu B^{*} \mu C^{*}-\int_{C^{*} \times B^{*}} h+\int_{D^{*} \times B^{*}} h-\alpha \mu D^{*} \mu B^{*} \\
& =\int_{D^{*} \times B^{*}}(h(x, y)-\alpha) d x d y+\int_{C^{*} \times B^{*}}(\alpha-h(x, y)) d x d y \\
& \leqslant \int_{A^{*} \times B^{*}}|h(x, y)-\alpha| d x d y .
\end{aligned}
$$


5D. LEMmA. If $A \subseteq \omega, B_{i} \subseteq \omega$ for $i<k$, and $J \subseteq k$, where $k<\omega$, then

$$
\lim _{n \rightarrow \mathscr{F}} \frac{1}{\#\left(I_{n}\right)} \sum_{a \in I_{n} \cap A} \prod_{j<k} q\left(B_{j}, a\right) \prod_{j \in k \backslash J} \mu B_{j}^{*}=\int_{A^{*} \times \prod_{j<k} B_{j}^{*}} \prod_{j \in J} h\left(x, y_{j}\right) d x d \mathbf{y}
$$

Proof. Since we can extract a factor $\prod_{j \in k \backslash J} \mu B_{j}^{*}$ from both expressions, it is enough to consider the case $J=k$.

(a) Note first that if $\alpha \in[0,1]^{k}$ then

$$
\begin{aligned}
& \left|\lim _{n \rightarrow \mathscr{F}} \frac{1}{\#\left(I_{n}\right)} \sum_{a \in I_{n} \cap A} \prod_{j<k} q\left(B_{j}, a\right)-\int_{A^{*} \times \prod_{j<k} B_{j}^{*}} \prod_{j<k} h\left(x, y_{j}\right) d x d \mathbf{y}\right| \\
& \leqslant 2 \sum_{j<k}\left(\int_{A^{*} \times B_{j}^{*}}\left|h(x, y)-\alpha_{j}\right| d x d y \cdot \prod_{\substack{l<k \\
l \neq j}} \mu B_{l}^{*}\right) .
\end{aligned}
$$

To see this, first compute

$$
\begin{aligned}
\lim _{n \rightarrow \mathscr{F}} & \frac{1}{\#\left(I_{n}\right)} \sum_{a \in I_{n} \cap A} \prod_{j<k} q\left(B_{j}, a\right)-\mu A^{*} \prod_{j<k} \alpha_{j} \mu B_{j}^{*} \mid \\
& \leqslant \lim _{n \rightarrow \mathscr{F}} \frac{1}{\#\left(I_{n}\right)} \sum_{a \in I_{n} \cap A}\left|\prod_{j<k} q\left(B_{j}, a\right)-\prod_{j<k} \alpha_{j} \mu B_{j}^{*}\right| \\
& \leqslant \lim _{n \rightarrow \mathscr{F}} \frac{1}{\#\left(I_{n}\right)} \sum_{a \in I_{n} \cap A} \sum_{j<k}\left|q\left(B_{j}, a\right)-\alpha_{j} \mu B_{j}^{*}\right| \prod_{\substack{l<k \\
l \neq j}} \mu B_{l}^{*} \\
& \leqslant \sum_{j<k} \prod_{\substack{l<k \\
l \neq j}} \mu B_{l}^{*} \int_{A^{*} \times B_{j}^{*}}\left|h(x, y)-\alpha_{j}\right| d x d y \quad(\text { by } 5 \mathrm{C}) .
\end{aligned}
$$

On the other hand,

$$
\begin{aligned}
& \left|\mu A^{*} \prod_{j<k} \alpha_{j} \mu B_{j}^{*}-\int_{A^{*} \times \prod_{j<k} B_{j}^{*}} \prod_{j<k} h\left(x, y_{j}\right) d x d \mathbf{y}\right| \\
& \quad \leqslant \int_{A^{*} \times \Pi_{j<k} B_{j}^{*}}\left|\prod_{j<k} \alpha_{j}-\prod_{j<k} h\left(x, y_{j}\right)\right| d x d \mathbf{y} \\
& \quad \leqslant \sum_{j<k} \int_{A^{*} \times \Pi_{j<k} B_{j}^{*}}\left|\alpha_{j}-h\left(x, y_{j}\right)\right| d x d \mathbf{y} \quad \text { (by 5B) } \\
& \quad=\sum_{j<k} \prod_{\substack{l<k \\
l \neq j}} \mu B_{l}^{*} \int_{A^{*} \times B_{j}^{*}}\left|\alpha_{j}-h(x, y)\right| d x d y .
\end{aligned}
$$

Putting these together, we obtain the result. 
(b) Now suppose that $\left\langle C_{r}\right\rangle_{r<m}$ is a partition of $\omega$ and that $\alpha \in[0,1]^{m \times m}$. Then

$$
\begin{aligned}
& \left|\lim _{n \rightarrow \mathscr{F}} \frac{1}{\#\left(I_{n}\right)} \sum_{a \in I_{n} \cap A} \prod_{j<k} q\left(B_{j}, a\right)-\int_{A^{*} \times \Pi_{j<k} B_{j}^{*}} \prod_{j<k} h\left(x, y_{j}\right) d x d \mathbf{y}\right| \\
& \leqslant \sum_{\substack{s<m \\
\mathbf{r} \in m^{k}}} \mid \lim _{n \rightarrow \mathscr{F}} \frac{1}{\#\left(I_{n}\right)} \sum_{a \in I_{n} \cap A \cap C_{s}} \prod_{j<k} q\left(B_{j} \cap C_{r_{j}}, a\right) \\
& -\int_{\left(A \cap C_{s}\right)^{*} \times \Pi_{j<k}\left(B_{j} \cap C_{r_{j}}\right)^{*}} \prod_{j<k} h\left(x, y_{j}\right) d x d \mathbf{y} \\
& \leqslant 2 \sum_{\substack{s<m \\
\mathbf{r} \in m^{k}}} \sum_{j<k}\left(\int_{\left(A \cap C_{s}\right)^{*} \times\left(B_{j} \cap C_{r_{j}}\right)^{*}}\left|h(x, y)-\alpha_{s r_{j}}\right| d x d \mathbf{y}\right. \\
& \left.\cdot \prod_{\substack{l<k \\
l \neq j}} \mu\left(B_{l} \cap C_{r_{l}}\right) *\right) \\
& \leqslant 2 \sum_{j<k} \sum_{\substack{s<m \\
\mathbf{r} \in m^{k}}} \int_{C_{s}^{*} \times C_{r_{j}}^{*}}\left|h(x, y)-\alpha_{s r_{j}}\right| d x d y \cdot \prod_{\substack{l<k \\
l \neq j}} \mu C_{r_{l}}^{*} \\
& =2 \sum_{j<k} \sum_{\substack{s<m \\
r<m}} \int_{C_{s}^{*} \times C_{r}^{*}}\left|h(x, y)-\alpha_{s r}\right| d x d y \\
& =2 k \int\left|h(x, y)-\sum_{r, s<m} \alpha_{s r} \chi C_{s}^{*}(x) \chi C_{r}^{*}(y)\right| d x d y .
\end{aligned}
$$

But this can be made as small as we please by choosing $m, C_{r}, \alpha_{s r}$ carefully (because every measurable subset of $\omega^{*}$ is approximable by sets of the form $C^{*}$, by the last remark in $2 \mathrm{~A}$ ). So we have our result.

5E. LemmA. If $A \subseteq \omega, B_{j} \subseteq \omega$ for $j<k, J \subseteq k$, and $f: \omega^{k} \rightarrow \mathbf{R}$ is integrable, where $k<\omega$, then

$$
\int_{A^{*} \times \Pi_{j<k} B_{j}^{*}} \prod_{j \in J} h\left(x, y_{j}\right) f(\mathbf{y}) d x d \mathbf{y}=\lim _{n \rightarrow \mathscr{F}} \frac{1}{\#\left(I_{n}\right)} \sum_{a \in I_{n} \cap A} \int_{\Pi_{j<k} B_{j}^{\prime}(a)^{*}} f(\mathbf{y}) d \mathbf{y},
$$

where $B_{j}^{\prime}(a)=B_{j} \cap \Gamma[\{a\}]$ if $j \in J, B_{j}$ if $j \in k \backslash J$.

Proof. If $f$ is identically 1 , this is 5D. If $f$ is of the form $\chi\left(\Pi_{-j<k} C_{j}^{*}\right)$ it follows on replacing $B_{j}$ by $B_{j} \cap C_{j}$. Since both expressions are linear and \|\|$_{1}$-continuous in $f$, they are equal for all integrable $f$.

5F. THEOREM.

$$
\lambda_{h}\left(T_{\Delta}\right)=\lim _{n_{0} \rightarrow \mathscr{F}} \cdots \lim _{n_{p} \rightarrow \mathscr{F}} \frac{\#\left(\left\{\sigma: \sigma \in \prod_{r \leqslant p} I_{n_{r}}, \sigma \rightarrow(\Delta) \subseteq \Gamma\right\}\right)}{\prod_{r \leqslant p} \#\left(I_{n_{r}}\right) .}
$$

PROOF. For $k<\omega$ set

$$
F_{k}=\left\{\sigma: \sigma \in \omega^{k}, \sigma \rightarrow\left(\Delta \cap k^{2}\right) \subseteq \Gamma\right\},
$$


and for $\sigma \in F_{k}, i<\omega$ set

$$
B(\sigma, i)=\omega \cap \bigcap_{\substack{j<k \\(j, i) \in \Delta}} \Gamma[\{\sigma(j)\}] .
$$

For $\sigma \in \bigcup_{k<\omega} \omega^{k}$ define $\beta(J) \in[0,1]$ as follows. If $\sigma \in \omega^{k}$ where $k \geqslant p+1$, say

$$
\beta(\sigma)= \begin{cases}1 & \text { if } \sigma^{\rightarrow}(\Delta) \subseteq \Gamma, \\ 0 & \text { otherwise. }\end{cases}
$$

For other $\sigma$, define $\beta(\sigma)$ by downwards induction on $\#(\sigma)$, by taking

$$
\beta(\sigma)=\lim _{n \rightarrow \mathscr{F}} \frac{1}{\#\left(I_{n}\right)} \sum_{a \in I_{n}} \beta(\sigma \frown a) .
$$

(Compare $g_{\sigma}^{\Delta}$ in $2 \mathrm{H}$; as in $2 \mathrm{H}$, this last formula is valid for all $\sigma$.) Thus $\beta(\varnothing)$ is the value of the right-hand expression in the statement of this theorem.

Now define $\gamma: \bigcup_{k<\omega} \omega^{k} \rightarrow[0,1]$ by writing $\gamma(\sigma)=0$ if $\sigma \in \omega^{k} \backslash F_{k}$ and

$$
\begin{aligned}
& \gamma(\sigma)=1 \quad \text { if } \sigma \in F_{k} \text { and } k>p, \\
& \gamma(\sigma)=\int_{\prod_{k \leqslant l \leqslant p} B(\sigma, l)^{*}} \prod_{\substack{k \leqslant l<m \leqslant p \\
(l, m) \in \Delta}} h\left(x_{l}, x_{m}\right) d x_{k} \cdots d x_{p}
\end{aligned}
$$

if $\sigma \in F_{k}$ and $k \leqslant p$. Then we see that if $k<p$ and $\sigma \in F_{k}$,

$$
\lim _{n \rightarrow \mathscr{F}} \frac{1}{\#\left(I_{n}\right)} \sum_{a \in I_{n}} \gamma(\sigma \frown a)=\lim _{n \rightarrow \mathscr{F}} \frac{1}{\#\left(I_{n}\right)} \sum_{a \in I_{n} \cap B(\sigma, k)} \gamma(\sigma \frown a)=\gamma(\sigma),
$$

on applying Lemma $5 \mathrm{E}$ with $A=B(\sigma, k), B_{j}=B(\sigma, j)$ for $k<j \leqslant p, J=\{j$ : $k<j \leqslant p,(k, j) \in \Delta\}, x=x_{k}, y_{j}=x_{j}$ for $k<j \leqslant p$, and

$$
f(\mathbf{y})=\prod_{\substack{k<l<m \leqslant p \\(l, m) \in \Delta}} h\left(y_{l}, y_{m}\right) \text {. }
$$

The formula

$$
\gamma(\sigma)=\lim _{n \rightarrow \mathscr{F}} \frac{1}{\#\left(I_{n}\right)} \sum_{a \in I_{n}} \gamma(\sigma \frown a)
$$

is valid if $\sigma \in F_{p}$, as then

$$
\gamma(\sigma)=\mu B(\sigma, p)^{*}=\lim _{n \rightarrow \mathscr{F}} \frac{1}{\#\left(I_{n}\right)} \sum_{a \in I_{n} \cap B(\sigma, p)} 1,
$$

and it is also valid for $\sigma \in \omega^{k} \backslash F_{k}$, as then both sides are zero. So as $\gamma(\sigma)=\beta(\sigma)$ for $\sigma \in \omega^{p+1}, \gamma(\sigma)=\beta(\sigma)$ for all $\sigma$, and $\gamma(\varnothing)=\beta(\varnothing)$. On the other hand, $B(\varnothing, l)=\omega$ for every $l \leqslant p$, so

$$
\gamma(\varnothing)=\int_{\omega^{* p+1}} \prod_{(l, m) \in \Delta} h\left(x_{l}, x_{m}\right) d x_{0} \cdots d x_{p}=\lambda_{h}\left(T_{\Delta}\right),
$$

which proves the result. 
5G. TheOREM. Let $\nu \in M_{1}\left(\mathscr{P} \omega^{2 \uparrow}\right)$ be deletion-invariant. Let $\left\langle I_{n}\right\rangle_{n<\omega}$ be a sequence in $[\omega]^{<\omega}$ as in $2 \mathrm{H}$, and $\mathscr{F}$ any nonprincipal ultrafilter on $\omega$. Construct $\mu$ from $\left\langle I_{n}\right\rangle_{n<\omega}$ and $\mathscr{F}$ as in $2 \mathrm{I}$ and $5 \mathrm{~A}(\mathrm{iii})$, and for each $\Gamma \subseteq \omega^{2 \uparrow}$ construct $h(\Gamma): \omega^{* 2} \rightarrow[0,1]$ and $\lambda_{h(\Gamma)} \in M_{1}\left(\mathscr{P} \omega^{2 \uparrow}\right)$ as in $5 \mathrm{~A}(\mathrm{v})$, (vi). Then

$$
\nu=\int \lambda_{h(\Gamma)} \nu(d \Gamma)
$$

in the sense that $\nu\left(T_{\Delta}\right)=\int \lambda_{h(\Gamma)}\left(T_{\Delta}\right) \nu(d \Gamma)$ for every finite $\Delta \subseteq \omega^{2 \uparrow}$.

Proof. Take $p<\omega$ and $\Delta \subseteq p^{2 \uparrow}$. By the choice of $\left\langle I_{n}\right\rangle_{n<\omega}$,

$$
\lim _{n_{0} \rightarrow \infty} \cdots \lim _{n_{p} \rightarrow \infty} \frac{\#\left\{\sigma: \sigma \in \prod_{i \leqslant p} I_{n_{i}}, \sigma \rightarrow(\Delta) \subseteq \Gamma\right\}}{\prod_{i \leqslant p} \#\left(I_{n_{i}}\right)}
$$

exists for almost all $\Gamma$. But this limit (when it exists) is just $\lambda_{h(\Gamma)}\left(T_{\Delta}\right)$, by $5 F$. So by Lebesgue's theorem

$$
\int \lambda_{h(\Gamma)}\left(T_{\Delta}\right) \nu(d \Gamma)=\lim _{n_{0} \rightarrow \infty} \cdots \lim _{n_{p} \rightarrow \infty} \frac{\sum_{\sigma \in \prod_{i \leqslant p} I_{n_{i}}} \nu\left(T_{\sigma \rightarrow(\Delta)}\right)}{\prod_{i \leqslant p} \#\left(I_{n_{i}}\right)}=\nu\left(T_{\Delta}\right)
$$

because $\nu$ is deletion-invariant.

5H. THEOREM. Let $\nu \in M_{1}\left(\mathscr{P} \omega^{2 \uparrow}\right)$. Then the following are equivalent:

(i) $\nu$ is expressible as $\lambda_{h}$ for some probability space $(X, \mu)$ and some measurable $h$ : $X^{2} \rightarrow[0,1]$

(ii) $\nu$ is an extreme point of the set of deletion-invariant members of $M_{1}\left(\mathscr{P} \omega^{2 \uparrow}\right)$;

(iii) $\nu$ is deletion-invariant and $\nu\left(T_{\Delta \cup \Delta^{\prime}}\right)=\nu\left(T_{\Delta}\right) \nu\left(T_{\Delta^{\prime}}\right)$ whenever $\Delta$ and $\Delta^{\prime}$ are finite subsets of $\omega^{2 \uparrow}$ and $V(\Delta) \cap V\left(\Delta^{\prime}\right)=\varnothing$.

Proof. (a) Write $C$ for the set of deletion-invariant members of $M_{1}\left(\mathscr{P} \omega^{2 \uparrow}\right)$; then $C$ is a compact convex subset of $M_{1}\left(\mathscr{P} \omega^{2 \uparrow}\right)$. If $\nu$ is an extreme point of $C$, then, by $5 \mathrm{G}, \nu$ has an expression in the form

$$
\nu=\int \lambda_{h(\Gamma)} \nu(d \Gamma)
$$

But since every $\lambda_{h(\Gamma)}$ belongs to $C$, we must have $\nu=\lambda_{h(\Gamma)}$ for almost all $\Gamma$. Thus (ii) $\Rightarrow$ (i).

(b) (i) $\Rightarrow$ (iii) is an elementary calculation.

(c) Finally, suppose that $\nu \in C$ but is not an extreme point of $C$. Then we can find $\nu_{1}, \nu_{2} \in C$ and a finite $\Delta \subseteq \omega^{2 \uparrow}$ such that $\nu=\frac{1}{2}\left(\nu_{1}+\nu_{2}\right)$ and $\nu_{1}\left(T_{\Delta}\right) \neq \nu_{2}\left(T_{\Delta}\right)$. Let $\Delta^{\prime}$ be such that $\Delta^{\prime}$ is expressible as $f^{\rightarrow}(\Delta)$ for some $f \in \omega^{\omega \uparrow}$, and $V(\Delta) \cap V\left(\Delta^{\prime}\right)=\varnothing$. Because (ii) $\Rightarrow$ (iii), we must have

$$
\nu_{i}\left(T_{\Delta \cup \Delta^{\prime}}\right) \geqslant \nu_{i}\left(T_{\Delta}\right) \nu_{i}\left(T_{\Delta^{\prime}}\right)=\nu_{i}\left(T_{\Delta}\right)^{2}
$$

for both $i$. So $\nu\left(T_{\Delta \cup \Delta^{\prime}}\right)>\nu\left(T_{\Delta}\right) \nu\left(T_{\Delta^{\prime}}\right)$ and $\nu$ does not satisfy (iii). Thus (iii) $\Rightarrow$ (ii). 


\section{A refinement of $4 A$ and $4 B$.}

We show how different, and essentially simpler, methods can be used to solve another problem from [1] (6D) and give an alternative proof of Proposition 4A.

6A. Lemma. Suppose that $\eta>0$ and that $0<\eta^{\prime}<\eta^{2}$. Then there is an integer $m$ such that whenever $(X, \mu)$ is a probability space and $\left\langle E_{i}\right\rangle_{i<m}$ a finite sequence of measurable sets with $\mu E_{i} \geqslant \eta$ for every $i<m$, there are $i<j<m$ such that $\mu\left(E_{i} \cap E_{j}\right) \geqslant \eta^{\prime}$.

Proof. This is a special case of the result at the bottom of p. 110 in [1].

6B. Corollary. Let $(X, \mu)$ be a probability space and $\left\langle E_{i j}\right\rangle_{i<j<\omega}$ a family of measurable subsets of $X$ such that $\inf _{i<j<\omega} \mu E_{i j} \geqslant \eta>0$. Suppose that $0<\eta^{\prime}<\eta^{2}$. Then there is an infinite $B \subseteq \omega$ such that

$$
\mu\left(E_{l j} \cap E_{i j}\right) \geqslant \eta^{\prime}, \quad \mu\left(E_{k l} \cap E_{i j}\right) \geqslant \eta^{\prime}
$$

whenever $k<l<i<j$ in $B$.

Proof. By Ramsey's theorem there is an infinite $B \subseteq \omega$ such that either

(i) $\mu\left(E_{l j} \cap E_{i j}\right)<\eta^{\prime}$ whenever $l<i<j$ in $B$, or

(ii) $\mu\left(E_{k l} \cap E_{i j}\right)<\eta^{\prime}$ whenever $k<l<i<j$ in $B$, or

(iii) $\mu\left(E_{l j} \cap E_{i j}\right) \geqslant \eta^{\prime}$ and $\mu\left(E_{k l} \cap E_{i j}\right) \geqslant \eta^{\prime}$ whenever $k<l<i<j$ in $B$.

But let $m<\omega$ be given by Lemma 6A. Then in case (i) there is a $j \in B$ such that $\mu(B \cap j)=m$ and we get a contradiction to $6 \mathrm{~A}$ on setting $E_{i}=E_{i j}$ for $i \in B \cap j$. While in case (ii) we enumerate $B$ in ascending order as $\langle r(i)\rangle_{i<\omega}$ and obtain a contradiction to $6 \mathrm{~A}$ on setting $E_{i}=E_{r(2 i), r(2 i+1)}$ for $i<m$. Thus we are left with case (iii), which is the required situation.

6C. TheOREM. Let $\nu \in M_{1}\left(\mathscr{P} \omega^{2 \uparrow}\right)$, and suppose that $w(\nu)>0$. Then there is an infinite $A \subseteq \omega$ such that $\nu\left(T_{\Delta}\right)>0$ whenever $\Delta \subseteq A^{2 \uparrow}$ is finite and there are no $i, j, k$ such that both $(i, j)$ and $(j, k)$ belong to $\Delta$.

Proof. For $I, J \subseteq \omega$ set

$$
G_{I J}=\left\{\Gamma: \Gamma \subseteq \omega^{2 \uparrow},(i, j) \in \Gamma \text { whenever } j \in J, i \in I \cap j\right\} .
$$

We seek to define, by induction, sequences $\langle\sigma(n)\rangle_{n<\omega},\left\langle B_{n}\right\rangle_{n<\omega}$ and $\left\langle\eta_{n}\right\rangle_{n<\omega}$ such that

$$
\begin{aligned}
& \sigma(n)<\omega, \quad B_{n} \in[\omega]^{\omega}, \quad \eta_{n}>0, \quad \sigma(r)<\min B_{n} \quad \forall r<n, \\
& \nu G_{I \cup\{i\}, J \cup\{j\}} \geqslant \eta_{n} \quad \text { whenever } I \subseteq K_{n}, J=K_{n} \backslash I \text { and }(i, j) \in B_{n}^{2 \uparrow},
\end{aligned}
$$

for every $n<\omega$, where $K_{n}=\{\sigma(r): r<n\}$. We start the induction by setting $\eta_{0}=w(\nu)$ and $B_{0}=\omega$. For the inductive step, take $\left.\eta_{n+1} \in\right] 0, \eta_{n}^{2}[$. Apply $6 \mathrm{~B}$ repeatedly to find an infinite $C \subseteq B_{n}$ such that

$$
\begin{aligned}
& \nu\left(G_{I \cup\{l\}, J \cup\{j\}} \cap G_{I \cup\{i\}, J \cup\{j\}}\right) \geqslant \eta_{n+1}, \\
& \nu\left(G_{I \cup\{k\}, J \cup\{l\}} \cap G_{I \cup\{i\}, J \cup\{j\}}\right) \geqslant \eta_{n+1}
\end{aligned}
$$


whenever $I \subseteq K_{n}, J=K_{n} \backslash I$, and $k<l<i<j$ in $C$. Take $k$ to be the least member of $C, \sigma(n)$ to be the least member of $C \backslash\{k\}$, and $B_{n+1}=C \backslash\{k, \sigma(n)\}$.

Suppose that $I \subseteq K_{n+1}, J=K_{n+1} \backslash I$, and $(i, j) \in B_{n+1}^{2 \uparrow}$. Then $\sigma(n)$ belongs to exactly one of $I, J$. (i) If $\sigma(n) \in I$, then write $I^{\prime}=I \backslash\{\sigma(n)\}$; now

$$
\nu G_{I \cup\{i\}, J \cup\{j\}}=\nu\left(G_{I^{\prime} \cup\{\sigma(n)\}, J \cup\{j\}} \cap G_{I^{\prime} \cup\{i\}, J \cup\{j\}}\right) \geqslant \eta_{n+1} .
$$

(ii) If $\sigma(n) \in J$, then write $J^{\prime}=J \backslash\{\sigma(n)\}$; now

$$
\nu G_{I \cup\{i\}, J \cup\{j\}} \geqslant \nu\left(G_{I \cup\{k\}, J^{\prime} \cup\{\sigma(n)\}} \cap G_{I \cup\{i\}, J^{\prime} \cup\{j\}}\right) \geqslant \eta_{n+1} .
$$

Thus the induction continues.

On completing the induction, set $A=\{\sigma(n): n<\omega\}$. If $\Delta \subseteq A^{2 \uparrow}$ is finite and $\{(0,1),(1,2)\} \nsubseteq \subset \Delta$, let $n$ be such that $V(\Delta) \subseteq K_{n}$ and set $I=\pi_{1}[\Delta], J=K_{n} \backslash I$. Then $\Delta \subseteq(I \times \vec{J}) \cap \omega^{2 \uparrow}$ so $T_{\Delta} \supseteq G_{I J}$ and $\nu\left(T_{\Delta}\right) \geqslant \eta_{n}>0$.

6D. CoROllaRY. Let $\left\langle E_{i j}\right\rangle_{i<j<\omega}$ be a family of measurable subsets of $[0,1]$ such that $\inf _{i<j} \mu_{L} E_{i j}>0$. Then there is an infinite $A \subseteq \omega$ such that $\bigcap_{(i, j) \in \Delta} E_{i j} \neq \varnothing$ whenever $\Delta \subseteq A^{2 \uparrow}$ and there are no $i, j, k$ such that both $(i, j)$ and $(j, k)$ belong to $\Delta$.

Proof. There are compact sets $F_{i j} \subseteq E_{i j}$ such that $\mu_{L} F_{i j} \geqslant \frac{1}{2} \mu_{L} E_{i j}$ for all $i<j$. Define $\nu \in M_{1}\left(\mathscr{P} \omega^{2 \uparrow}\right)$ by writing

$$
\nu\left(T_{\Delta}\right)=\mu_{L}\left(\bigcap_{(i, j) \in \Delta} F_{i j}\right) \quad \forall \Delta \subseteq \omega^{2 \uparrow}
$$

in (b) of the proof of $1 \mathrm{E}$, and apply 6C to find $A \subseteq \omega$. Then if $\Delta \subseteq A^{2 \uparrow}$ and $\{(0,1)$, $(1,2)\} \not \subset \Delta, \nu\left(T_{\Delta^{\prime}}\right)>0$ for every finite $\Delta^{\prime} \subseteq \Delta$, so $\bigcap_{(i, j) \in \Delta^{\prime}} F_{i j} \neq \varnothing$ for every finite $\Delta^{\prime} \subseteq \Delta$, and (because the $F_{i j}$ are compact) $\bigcap_{(i, j) \in \Delta} F_{i j} \neq \varnothing$, so $\bigcap_{(i, j) \in \Delta} E_{i j} \neq \varnothing$.

REMARK. Of course there is no need to speak of measures on $\mathscr{P} \omega^{2 \uparrow}$ in proving this corollary; we express Theorem $6 \mathrm{C}$ in this language only to maintain links with the rest of the paper. $6 \mathrm{D}$ answers the last question of $\S 3$ of [1].

7. Concluding remarks. We claimed to have solved "nearly all" the questions left open by [1] which referred to graphs on $\omega$; it behoves us to be explicit about the one we have not answered, 7A. We follow this with a description of further questions of our own which we have not so far resolved.

7A. $A$ refinement of $1 \mathrm{~A}(\mathrm{f})$. Suppose that $\nu \in M_{1}\left(\mathscr{P} \omega^{2 \uparrow}\right)$ and that $\Delta \subseteq \omega^{2 \uparrow}$ is finite. Set

$$
\varphi(\Delta, \nu)=\sup \left\{\nu\left(T_{f \rightarrow(\Delta)}\right): f \in \omega^{\omega \uparrow}\right\}
$$

and for $u \in[0,1]$ set

$$
\psi_{\Delta}(u)=\inf \left\{\varphi(\Delta, \nu): \nu \in M_{1}\left(\mathscr{P} \omega^{2 \uparrow}\right), w(\nu) \geqslant u\right\} .
$$

In [1], the authors speculate on the value of $\psi_{\Delta}(u)$ for $\Delta=n^{2 \uparrow}$.

Of course $\psi_{\Delta}(u)=0$ if $u<u_{0}(\Delta)$. The machinery developed in this paper enables us to say very much more. Consider first the formula in the proof of $5 \mathrm{G}$ :

$$
\int \lambda_{h(\Gamma)}\left(T_{\Delta}\right) \nu(d \Gamma)=\lim _{n_{0} \rightarrow \infty} \cdots \lim _{n_{p} \rightarrow \infty} \frac{\sum_{\sigma \in \Pi_{i \leqslant p} I_{n_{i}}} \nu\left(T_{\sigma \rightarrow(\Delta)}\right)}{\prod_{i \leqslant p} \#\left(I_{n_{i}}\right)},
$$


which is valid for any $\nu \in M_{1}\left(\mathscr{P} \omega^{2 \uparrow}\right)$. From this we see that (in the context of that proof)

$$
\int \lambda_{h(\Gamma)}\left(T_{\Delta}\right) \nu(d \Gamma) \leqslant \varphi(\Delta, \nu)
$$

At the same time, the $h(\Gamma)$ have been chosen in such a way that, for almost all $\Gamma \subseteq \omega^{2 \uparrow}$,

$$
w\left(\lambda_{h(\Gamma)}\right)=\lambda_{h(\Gamma)}\left(U_{01}\right)=\lim _{m \rightarrow \infty} \lim _{n \rightarrow \infty} \frac{\sum_{a \in I_{m}, b \in I_{n}} \nu\left(U_{a b}\right)}{\#\left(I_{m}\right) \#\left(I_{n}\right)} \geqslant w(\nu) .
$$

So we see that, for some $\Gamma \subseteq \omega^{2 \uparrow}$, we must have simultaneously

$$
w\left(\lambda_{h(\Gamma)}\right) \geqslant w(\nu), \quad \lambda_{h(\Gamma)}\left(T_{\Delta}\right) \leqslant \varphi(\Delta, \nu) .
$$

But as $\lambda_{h(\Gamma)}$ is deletion-invariant, $\lambda_{h(\Gamma)}\left(T_{\Delta}\right)=\varphi\left(\Delta, \lambda_{h(\Gamma)}\right)$.

Write $C$ for the set of deletion-invariant measures in $M_{1}\left(\mathscr{P} \omega^{2 \uparrow}\right)$, and $C^{\prime}$ for the set of $h$-representable measures (which we know to be the set of extreme points of $C$, by Theorem $5 \mathrm{H})$. The paragraph just past shows that

$$
\begin{aligned}
\psi_{\Delta}(u) & =\inf \left\{\varphi(\Delta, \nu): \nu \in C^{\prime}, w(\nu) \geqslant u\right\} . \\
& =\inf \left\{\nu\left(T_{\Delta}\right): \nu \in C, \nu\left(U_{01}\right) \geqslant u\right\} .
\end{aligned}
$$

Since $C$ is compact, this last infimum is attained. Consequently $\psi_{\Delta}(u)>0$ if $u>u_{0}(\Delta)$. Also, if $0 \leqslant u<u^{\prime} \leqslant 1$, then

$$
\psi_{\Delta}\left(u^{\prime}\right) \leqslant \frac{u^{\prime}-u}{1-u}+\frac{1-u^{\prime}}{1-u} \psi_{\Delta}(u)
$$

this can easily be seen by taking a $\nu$ such that $w(\nu)=u$ and $\varphi(\Delta, \nu) \simeq \psi_{\Delta}(u)$, and then calculating $w\left(\nu^{\prime}\right)$ and $\varphi\left(\Delta, \nu^{\prime}\right)$ where $\nu^{\prime}=\varepsilon \nu_{0}+(1-\varepsilon) \nu, \varepsilon=\left(u^{\prime}-u\right) /(1-u)$ and $\nu_{0} \in C$ is the point mass given by $\nu_{0}\left(\left\{\omega^{2 \uparrow}\right\}\right)=1$. This shows that $\psi_{\Delta}$ is continuous.

Erdös and Hajnal show that $\psi_{\Delta}(u) \geqslant \max (0, u(2 u-1))$ when $\Delta=3^{2 \uparrow}$, by special arguments. In view of the discussion above this simplifies to showing that if $(X, \mu)$ is a probability space and $h: X^{2} \rightarrow[0,1]$ is a measurable function with $\int h=u$, then

$$
\int h(x, y) h(x, z) h(y, z) d x d y d z \geqslant u(2 u-1),
$$

the arguments for which are given in [1]. More generally, they suggest the formula

$$
\psi_{\Delta}(u)=u(2 u-1)(3 u-2) \cdots((n-1) u-(n-2))
$$

if $\Delta=n^{2 \uparrow}$, where $n \geqslant 3$, and $u \geqslant u_{0}(\Delta)=(n-2) /(n-1)$. But we ourselves have no views on this matter.

7B. Other open questions. (a) Our experience with the algorithm described in $3 \mathrm{~L}$ is that in a great many cases it finds the critical relation, or relations, predicted by $3 \mathrm{D}$ quite quickly, and must then spend far more time to check that they are indeed best possible. (Thus the abbreviated descriptions in $3 \mathrm{M}$ do in fact give a fair idea of the balance between the two parts of the process.) We therefore ask whether our methods can be improved either (i) by finding a better bound than $2^{p-1}$ for the largest \#(V(H)) that needs to be considered, or (ii) sharpening $3 \mathrm{~K}$ to limit further the number of relations that need to be examined in our inductive constructions. 
(b) Our algorithm is certainly not polynomial-time, since it has to consider all simple improvements of certain matrices of sizes at least comparable to the size of the input $\Delta$. So we ask: is there a polynomial-time algorithm to calculate $u_{0}(\Delta)$ ?

(c) Set $\Delta_{A}=\{(i, j): i<j<\omega, i+j$ is odd $\}$. Then $u_{0}\left(\Delta_{A}\right)=\frac{1}{2}$ (4D). But we have no formula for $\alpha_{n}=u_{0}\left(\Delta_{A} \cap n^{2}\right)$. The values known to us are $\alpha_{2}=0, \alpha_{3}=\frac{1}{4}, \alpha_{4}=\frac{1}{3}$ (3M(a)), $\alpha_{5}=\frac{3}{8}, \alpha_{6}=\frac{3}{7}, \alpha_{7}=\frac{4}{9}, \alpha_{8} \geqslant \frac{11}{24}$.

(d) Set $\Delta=\{(i, j): i<j \leqslant i+2\}$. Is $u_{0}(\Delta)$ equal to $\frac{2}{3}$ ? More generally, we conjecture that if $\Delta\{(i, j): i<j \leqslant i+k\}$ then $u_{0}(\Delta)=k /(k+1)$.

(e) Precisely which numbers $u \in[0,1]$ can be (i) $v(H)$ for a finite relation $H$, (ii) $u_{0}(\Delta)$ for a finite $\Delta \subseteq \omega^{2 \uparrow}$, and (iii) $u_{0}(\Delta)$ for an arbitrary $\Delta \subseteq \omega^{2 \uparrow}$ ? 3E answers this for $u<\frac{1}{2}$; with $2 \mathrm{~J}$ and $3 \mathrm{M}$ we can extend the analysis to $u \leqslant \frac{4}{7}$. Above this level the situation seems to be very much more complicated.

\section{REFERENCES}

1. P. Erdös and A. Hajnal, Some remarks on set theory. IX. Combinatorial problems in measure theory and set theory, Michigan Math. J. 11 (1964), 107-127.

Department of Mathematics, University of Essex, Colchester, England

Departement de Mathematiques, Équipe d’Analyse, Université Paris Vi, Paris, France 